\title{
SLC22A5 (OCTN2) Carnitine Transporter-Indispensable for Cell Metabolism, a Jekyll and Hyde of Human Cancer
}

\author{
Barbara Juraszek and Katarzyna A. Nałęcz * \\ Laboratory of Transport through Biomembranes, Nencki Institute of Experimental Biology of Polish Academy \\ of Sciences, 3 Pasteur Street, 02-093 Warsaw, Poland; b.juraszek@nencki.edu.pl \\ * Correspondence: k.nalecz@nencki.edu.pl; Tel.: +48-22-5892303 \\ Academic Editors: Cesare Indiveri and Lara Console \\ Received: 20 November 2019; Accepted: 14 December 2019; Published: 19 December 2019

\begin{abstract}
Oxidation of fatty acids uses L-carnitine to transport acyl moieties to mitochondria in a so-called carnitine shuttle. The process of $\beta$-oxidation also takes place in cancer cells. The majority of carnitine comes from the diet and is transported to the cell by ubiquitously expressed organic cation transporter novel family member 2 (OCTN2)/solute carrier family 22 member 5 (SLC22A5). The expression of $S L C 22 A 5$ is regulated by transcription factors peroxisome proliferator-activated receptors (PPARs) and estrogen receptor. Transporter delivery to the cell surface, as well as transport activity are controlled by OCTN2 interaction with other proteins, such as PDZ-domain containing proteins, protein phosphatase PP2A, caveolin-1, protein kinase C. SLC22A5 expression is altered in many types of cancer, giving an advantage to some of them by supplying carnitine for $\beta$-oxidation, thus providing an alternative to glucose source of energy for growth and proliferation. On the other hand, SLC22A5 can also transport several chemotherapeutics used in clinics, leading to cancer cell death.
\end{abstract}

Keywords: carnitine; SLC22A5/OCTN2; cancer

\section{Introduction}

Fatty acids fulfill various roles in the cell: they are components of membrane lipids, they regulate proteins in lipid posttranslational modifications, they control signal transduction pathways and, last but not least, they constitute an energy source in oxidation.

In healthy humans, oxidation of fatty acids takes place mainly in liver, but it is also very active in energy-demanding tissues, e.g., skeletal muscle and heart. This is why disorders of fatty acid oxidation result in symptoms like myopathy and progressive cardiomyopathy. Other symptoms have also been reported, such as neuropathy, hypoglycemic hypoketotic encephalopathy or Reye-like syndrome, seizures and mental retardation [1], which points to an involvement of $\beta$-oxidation in the physiological activity of the brain.

At the molecular level, there exist two cellular compartments in which fatty acid oxidation occurs: peroxisomes and mitochondria. In peroxisomes, very-long-, branched-chain- and medium-chain fatty acids are oxidized (Figure 1) in the process of $\alpha$ - and $\beta$-oxidation (for review, see [2]). Long-chain fatty acids are oxidized inside mitochondria in the process of $\beta$-oxidation. In order to enter the oxidative pathway, fatty acids have to be activated to acylCoAs. Very-long chain fatty acids are activated on the cytoplasmic side of peroxisomes and are transported to these organelles by one of the transporters belonging to the ATP-binding cassette (ABC) subfamily D [3]. The mechanism of medium-chain fatty acids' entry into peroxisomes still remains obscure; they are activated inside these organelles at the expense of ATP, transported to peroxisomes by solute carrier (SLC)-SLC25A17 
(Ant1p/PMP3) transporter [4] in an exchange with AMP formed in the process of fatty acid activation. In the mitochondria, CoA derivatives of long-chain fatty acids are formed by acyl-CoA syntethase (ACLS), an enzyme containing one transmembrane domain and localized in the outer mitochondrial membrane. Since the CoA derivatives of fatty acids do not penetrate lipid bilayer, fatty acyl moiety is delivered to mitochondrial matrix thanks to the so-called carnitine shuttle (Figure 1).

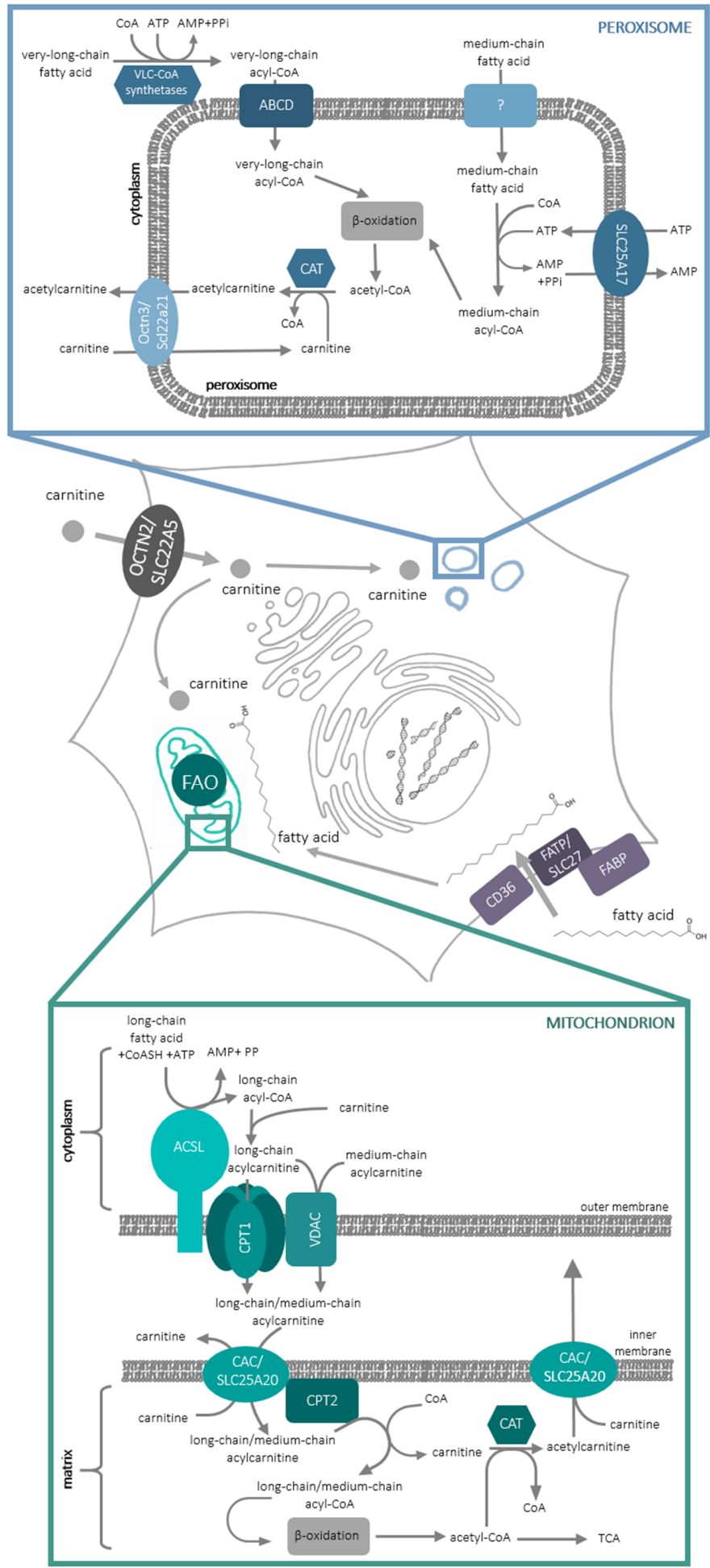

Figure 1. Fatty acid oxidation processes involving carnitine in mammalian cell. For a detailed description and abbreviations see the text. FAO, fatty acid oxidation. 
L-carnitine, (3R)-3-Hydroxy-4-(trimethylazaniumyl)butanoate, contains 3 functional groups: trimethylamine-, carboxyl- and a hydroxyl group, which is capable of forming an ester bond with acidic compounds. The acyl moiety of long-chain acyl CoA is transferred to this hydroxyl group of L-carnitine by carnitine palmitoyltransferase 1 (CPT1) protein, which is also situated in the outer mitochondrial membrane. CPT1 can be inhibited by malonyl-CoA, first intermediate in fatty acid synthesis, thus making this enzyme a control switch between fatty acid catabolism and synthesis. CPT1 contains 2 transmembrane domains, with its $\mathrm{N}$ - and C-terminus and both the malonyl-CoA binding site and the active site, on the cytoplasmic side of the enzyme [5]. Such a location of the active site requires a mechanism responsible for acylcarnitine transport to the mitochondrial matrix. It is possible that acylcarnitines may cross the outer mitochondrial membrane through the mitochondrial Voltage-dependent anion channel (VDAC) [6]. Since CPT1 forms dimers, which can further oligomerize into hexamers [7], it was also proposed that acylcarnitines can cross the outer mitochondrial membrane through CPT1 hexamer. It is noteworthy that CPT1 was shown to interact with both ACLS and VDAC [8]. Acyl-carnitines are further transported through the inner mitochondrial membrane by carnitine-acylcarnitine carrier (CAC/SLC25A20) in an exchange reaction with free L-carnitine leaving the mitochondrial matrix $[9,10]$. CAC interacts with carnitine palmitoyltransferase 2 (CPT2), which is located on the inner side of the inner mitochondrial membrane and releases free L-carnitine and acyl-CoA, which can enter the $\beta$-oxidation pathway (Figure 1). It needs to be added that carnitine acyl transferases are also present in the peroxisomes [11] and, since the oxidation of fatty acids in these organelles is not complete, it was suggested that shortened fatty acids in the form of acylcarnitines can be exported from peroxisomes for further oxidation in mitochondria [11]. Slc22a21 (Octn3) was argued to catalyze this reaction [12].

\section{Carnitine Transporters}

Since carnitine is such an indispensable compound, not only for the translocation of fatty acids through the membranes but also for the regulation of acylCoA/CoA ratio, cells need a continued supply of carnitine. Carnitine biosynthesis begins with the methylation of lisyl residues in proteins and is followed by protein lysosomal degradation and further synthesis from trimethyllysine, which engages several enzymes (for review see [13]). Interestingly, the activity of the last enzyme in this pathway- $\gamma$-butyrobetaine dioxygenase- was detected exclusively in rat and mouse liver as well as in rat testis, while it was not detectable in the brain [14]. In humans, the endogenous synthesis is, however, insufficient and approximately $75 \%$ of carnitine is sourced from food, mainly from red meat [15]. Being a water-soluble zwitterion, carnitine has to use transporting proteins to cross the biological membranes. In accordance with the current classification of solute carriers (SLCs), the carnitine transporters belong to three different families: SLC22, SLC25 and SLC6 (Table 1).

Table 1. Mammalian carnitine transporters.

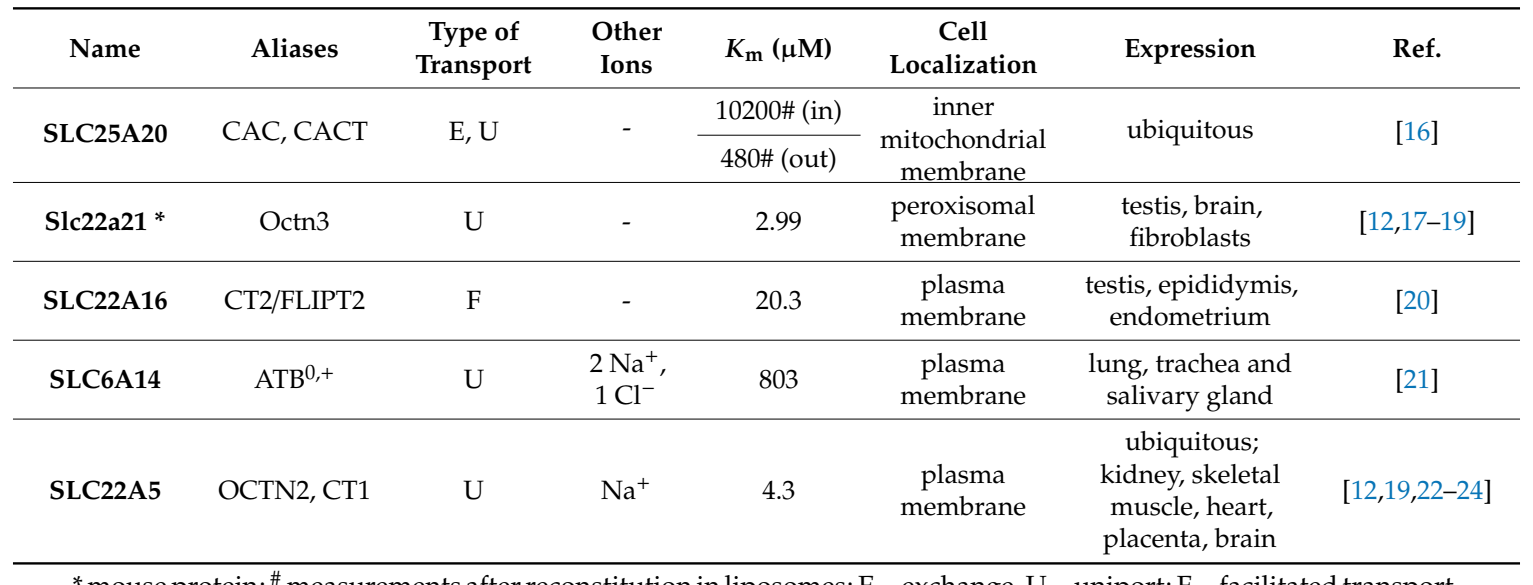

* mouse protein; ${ }^{\#}$ measurements after reconstitution in liposomes; E- exchange, U-uniport; F-facilitated transport. 
There are two carnitine transporters functioning in the intracellular membranes: SLC25A20 and Slc22a21. The first one is a carnitine carrier of the inner mitochondrial membrane [9,25] responsible for carnitine/acylcarnitine exchange (Figure 1). Its structure, consisting of six transmembrane helices, is typical of other mitochondrial carriers [10]. The second one, as mentioned in the Introduction, was detected in peroxisomes [12,18]. Although its gene (Slc22a21/Slc22a9) was cloned from mouse [17], the protein was also detected in human fibroblasts [18]. It is argued that the human gene is located at 5q31 between SLC22A4 and SLC22A5 and is associated with Crohn disease [26]. Octn3 was also proved to be present in the rat brain, in the peroxisomes of astrocytes [12]; moreover, its expression was up-regulated by treatment with peroxisome proliferators-activator receptor (PPAR) agonist [12].

There are three carnitine transporters that are situated in the plasma membrane and as such can supply carnitine to the cells: SLC22A16, SLC6A14 and SLC22A5. However, they all differ in their affinities for carnitine and their expression in the human body.

Carnitine transporter 2 (CT2/FLIPT2) coded by human SLC22A16 gene was identified [20] and it transports carnitine by facilitated diffusion. Although CT2 transports carnitine with a high affinity $\left(K_{\mathrm{m}}\right.$ $=20.3 \mu \mathrm{M})$, its expression is limited since it is mainly expressed in testis and epidydimis [20].

SLC6A14 is also capable of transporting carnitine, though with a very low affinity $\left(K_{\mathrm{m}}=\right.$ $830 \mu \mathrm{M}$ ) [21]. This is an amino acid transporter $\mathrm{ATB}^{0,+}$ specific for neutral (index " 0 ") and basic (index "+") amino acids and transports its substrate with two $\mathrm{Na}$ and one $\mathrm{Cl}$ ions [27]. Considering much lower $K_{\mathrm{m}}$ values for amino acids [27], the involvement of SLC6A14 in carnitine uptake seems less probable, although mice with inactive Slc22a5/Octn2 accumulated carnitine in the brain [28], which might arise from the presence of Slc6a14 in the blood-brain barrier [29,30].

Apart from transporting organic cations, SLC22A5/OCTN2-organic cation transporter novel family member 2 is characterized by a high affinity for carnitine $\left(K_{\mathrm{m}}=4.34 \mu \mathrm{M}\right)$ [22]. Its activity is inhibited by short- and long-chain acylcarnitines [22,31]; therefore, it was also named CT1 (carnitine transporter 1). The loss of OCTN2 function causes systemic carnitine deficiency [32]. The human gene coding SLC22A5 was cloned in 1998 [22,23] and the protein was named OCTN2 because of its similarity $(75.8 \%)$ to OCTN1/SLC22A4. Overexpressed OCTN1 was shown to transport carnitine in a $\mathrm{Na}^{+}$-dependent manner $[17,33]$; however, its activity was much lower in comparison with OCTN2 and OCTN3 [17]. Subsequently, OCTN1 was shown to be primarily an ergothioneine transporter [34].

What is more, SLC22A5/OCTN2 is ubiquitously expressed [22,23,31], which renders it the principal plasma membrane carnitine transporter. In addition, it is expressed in the brain; a transcriptome analysis using Affymetrix GeneChip Arrays demonstrated Slc22a5 expression in various brain cell types: astrocytes, neurons and oligodendrocytes [35]. The level of OCTN2 RNA expression was proved to increase during development in various brain regions [36]. It ought to be added that SLC22A5/OCTN2 was also detected in the brain at the protein level; it is located in the capillary endothelial cells forming the blood-brain barrier (Figure 2) [29], in astrocytes [12] and in neurons [24].

Recently, during the differentiation of human monocytes to macrophages, a possibility of carnitine transport by another amino acid transporter-SLC38A/SNAT2 was proposed, due to a correlation between an increased expression of this transporter and carnitine transport [37]. However, there is no conclusive evidence that would follow from an over-expressed transporter. 


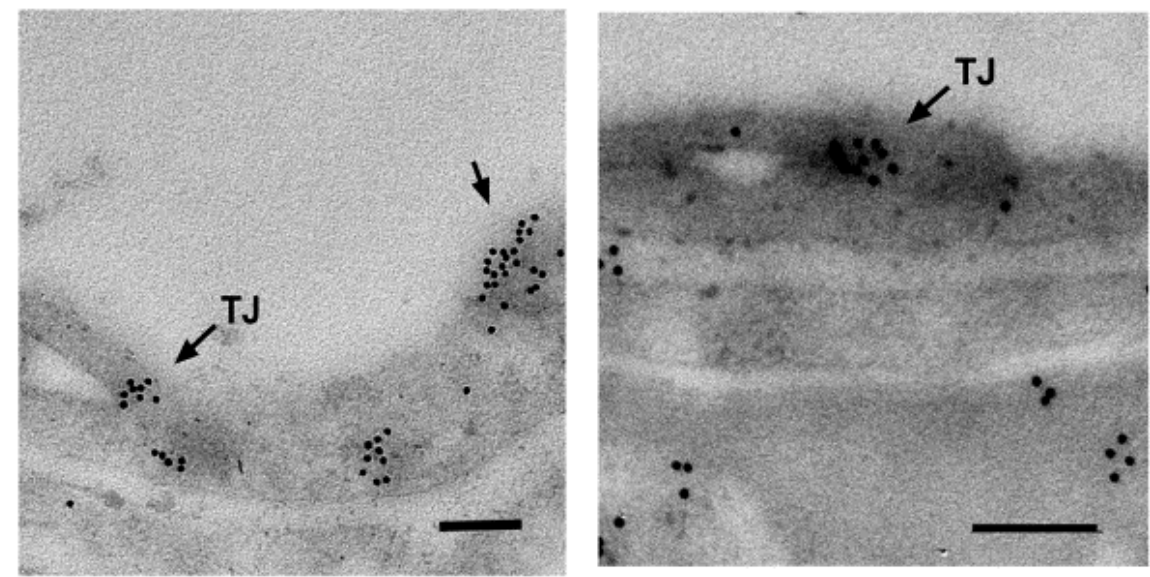

Figure 2. Presence of Octn2 in the blood-brain barrier. Rat brain slices were obtained, fixed and embedded in Epon after dehydration, as presented in [30]. They were subsequently treated with anti-OCTN2 antibody and the secondary antibody coupled to $10 \mathrm{~nm}$ gold particles, as presented in [12]. The areas with selected capillaries are shown. The TJ, tight junction; single arrow, Octn2 in the apical membrane. Octn2 is also detected in astrocytic endfeet (right panel). The bar size: $200 \mathrm{~nm}$.

\section{SLC22A5 Regulation}

SLC22A5/OCTN2 is the only high-affinity carnitine transporter that is located in the plasma membrane and is ubiquitously expressed. Therefore, it is strictly regulated-from transcription and translation-through posttranslational modifications and interactions with other proteins at various steps of protein trafficking to the plasma membrane (Figure 3).

Expression of SLC22A5 is regulated by estrogens, and an estrogen receptor responsive element was found in its first intron [38] —an interesting observation bearing in mind the role of estrogen signaling in breast cancer. SLC22A5 transcription is also significantly enhanced by agonists of nuclear receptors-peroxisome proliferator-activated receptors (PPARs) [39-42], (Figure 3A) and the PPAR $\alpha$ responsive element was also located to the first intron of $S L C 22 A 5$ [43]. This was confirmed for other species, including humans [44]. Furthermore, SLC22A5 expression was stimulated by pro-inflammatory cytokines, including tumor necrosis factor $\alpha(\mathrm{TNF} \alpha)$, and an involvement of a nuclear factor KB (NF-KB) was suggested [45]. What is more, during the differentiation of human monocytes to macrophages by granulocyte-macrophage colony stimulating factor, increased expression of SLC22A5 correlated with increased phosphorylation of mTOR kinase and activation of the transcription factor STAT3 [37].

The transcript of rat Slc22a5 was shown to be stabilized in endoplasmic reticulum (ER) through co-injection to Xenopus oocytes of cRNA coding cartregulin, a protein highly homologous to the first 146 amino acids of OCTN2 [46]. This co-expression also resulted in an elevated level of OCTN2 and an enhanced transport activity [46].

Being a plasma membrane protein, SLC22A5 is inserted co-translationally into the ER membrane, it is glycosylated in ER and Golgi at the first extracellular loop [47] and delivered in vesicular transport to the cell surface. This process was shown to be regulated by activation of protein kinase C (PKC) [48], although the transporter itself is not phosphorylated by this kinase [48,49]. At SLC22A5 C-terminus the last 4 amino acids STAF were proved to be motif binding proteins with so-called PDZ domains (named after proteins: postsynaptic density 95/disc large/zonula occludens-1). The pull-down experiments and immunochemistry analyses in the kidney brush-border membranes displayed co-localization of OCTN2 with PDZK1 $\left(\mathrm{Na}^{+} / \mathrm{H}^{+}\right.$exchange regulatory cofactor NHE-RF3), a phenomenon not observed after truncation of the last 4 amino acids [50]. Co-transfection with genes coding the both proteins led to a substantially enhanced transporting activity, without any change in OCTN2 surface presence. The same group revealed that co-expression of OCTN2 with PDZK2 (intestinal and kidney-enriched PDZ protein-IKEEPP, $\mathrm{Na}^{+} / \mathrm{H}^{+}$exchange regulatory cofactor NHE-RF4) resulted in the augmented transport activity and in the surface presence of the transporter [51]. A mass spectrometry analysis 
of SLC22A5 interactome in rat astrocytes allowed to identify, two PDZ proteins out of 156 proteins: zonula occludens-1 (ZO-1) and AHNAK [52]. In addition, experiments with overexpressed SLC22A5 exhibited co-localization with ZO-1 and no direct interaction with AHNAK [53], which can suggest that AHNAK may be a part of a bigger complex, especially because PDZ proteins are known to interact with one another (for review, see [54,55]). What is noteworthy, phosphorylation of ZO-1 by PKC as well as depletion of the STAF motif resulted in decreased carnitine transport [53], which led to a conclusion that non-phosphorylated ZO-1 maintains the transporter in its active state. The aforementioned observations point to the fact that the activity of SLC22A5 can be controlled by PDZ proteins (Figure 3B), similarly to the control of channel proteins [56].

D.

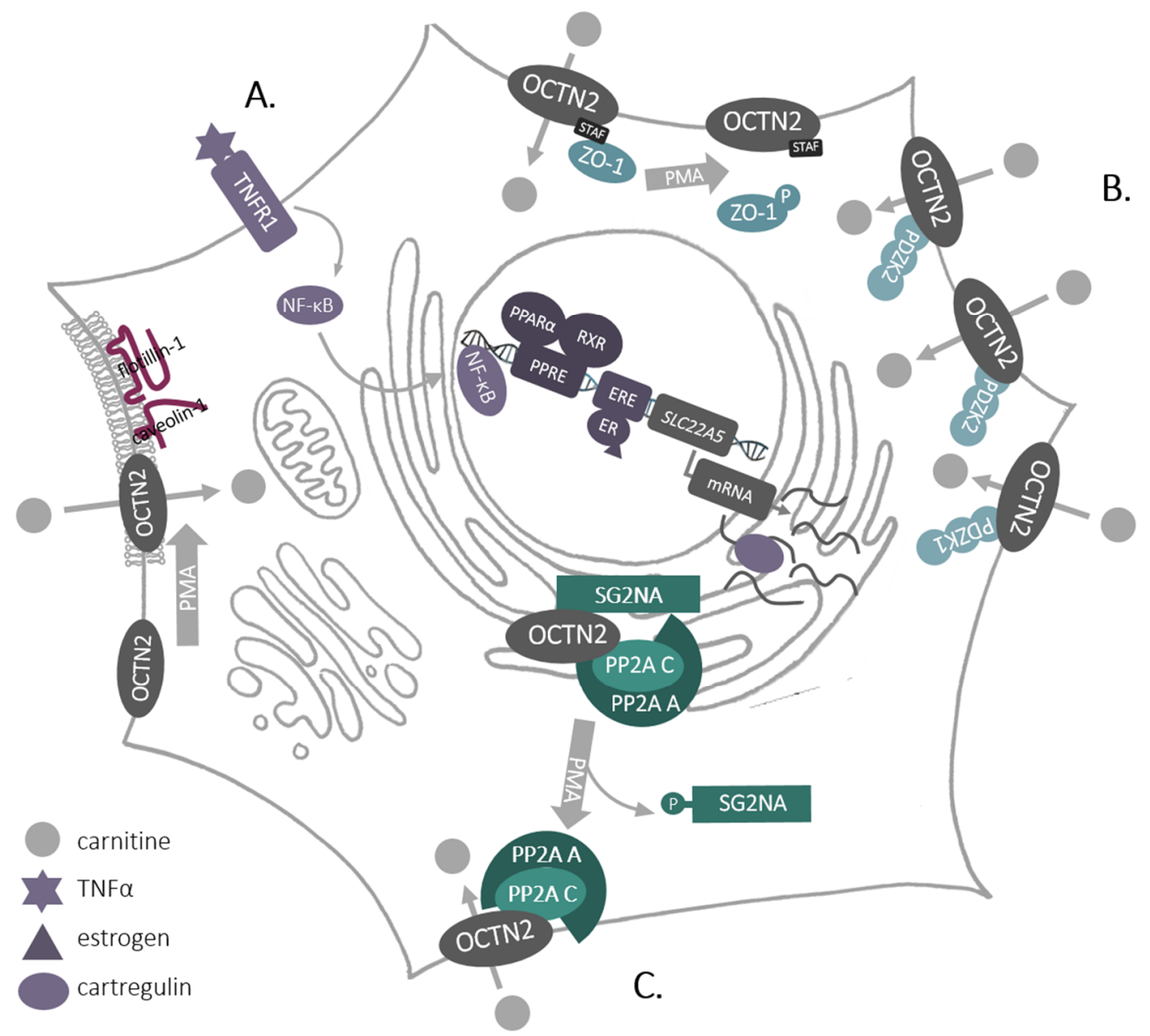

Figure 3. Regulation of SLC22A5/OCTN2 in the mammalian cell. A. Transcription of SLC22A5; B. Interaction of SLC22A5/OCTN2 with PDZ proteins; C. Trafficking to the plasma membrane-interaction with phosphatase PP2A; D. Lateral movement in the plasma membrane to rafts. For a detailed description and abbreviations see the text.

However, phosphorylation of ZO-1 did not affect the amount of SLC22A5 in the plasma membrane [53] and the trafficking of the transporter was shown to be controlled by PKC through protein phosphatase PP2A (Figure 3C), detected in SLC22A5 intaractome [52]. PP2A, as a complex containing structural subunit $A$, catalytic subunit $C$ and the regulatory subunit SG2NA, co-localizes with OCTN2 in the ER, while activation of PKC results in phosphorylation of the regulatory subunit SG2NA, leading to the transfer of the transporter with A and C PP2A subunits to the cell surface. This enables a carnitine uptake by the cell [52]. What is more, activation of PKC fosters a lateral movement of SLC22A5 in the plasma membrane, increasing the amount of the transporter in cholesterol-rich domains (rafts) and augmented interaction with caveolin-1 and flotillin-1 [48] (Figure 3D). Experiments with the deletion mutants proved a direct interaction between caveolin-1 and amino acids 14-22 and 
447-454 of rat Octn2 sequence [48]. All these observations demonstrate that SLC22A5 can be regulated by other proteins at various steps: exporting from the ER, trafficking to the plasma membrane and locating in microdomains, what affects transporter activity. Since activators of PKC, e.g., phorbol esters, are tumor promoters [57], while PP2A is considered to be a tumor suppressor [58], such comprehensive regulation of SLC22A5 may have implications in pathological states, in particular in cancer.

\section{SLC22A5 Pharmacological Implications}

Apart from carnitine, SLC22A5 can also transport organic cations, in a sodium-independent way [22] and pharmacologically active compounds [22,59]. Said compounds include e.g., verapamil, an anti-arhytmia agent [60], which is not surprising given the role of SLC22A5 and fatty acid oxidation in heart function. Interestingly, however, there are also clinically used anti-psychotic drugs (amisulpride [61]) and anti-cancer drugs such as etoposide [62], oxaliplatin [63] and imatinib [64] that are taken up by cells via SLC22A5 (Table 2). Even more drugs were proven to inhibit carnitine uptake by cells, acting as an SLC22A5 inhibitors (Table 3), including several anticancer drugs as well. Carnitine and its derivatives are believed to have neuroprotective properties; that is why there are several clinical trials underway studying whether carnitine can reduce neurotoxicity in cancer patients undergoing chemotherapy (https://clinicaltrials.gov/). Some researches established that even though there was no difference in overall peripheral neuropathy incidence between patients treated with combination of sagopilone and acetyl-L-carnitine and patients treated with sagopilone and placebo, the severity of this neuropathy was significantly lower in patients who were given Acetyl-L-carnitine [65]. On the other hand, it was revealed that Acetyl-L-carnitine in the long term could even increase taxane-induced neuropathy in women undergoing adjuvant breast cancer therapy [66]. This points to a need for a deeper understanding of the role played by carnitine, SLC22A5 and fatty acid oxidation in both cancer and in the brain-fields that up until recently have been under-researched. 
Table 2. Transport of drugs by OCTN2/SLC22A5.

\begin{tabular}{|c|c|c|c|c|c|c|c|c|}
\hline Drug & Drug Target and Use & & Conc. & Net Uptake & Time of Uptake & Type of Assay & Other Info & Ref. \\
\hline amisulpride & $\begin{array}{l}\text { selective dopamine antagonist; antipsychotic } \\
\text { drug, treatment of psychoses, schizophrenia } \\
\text { and persistent depressive disorder }\end{array}$ & hOCTN2 & $5 \mu \mathrm{M}$ & $\begin{array}{c}\approx 13 \pm 1.5 \\
\mathrm{pmol} / \mathrm{min} / \mathrm{mg} \\
\text { protein }\end{array}$ & $2 \mathrm{~min}$ & HPLC & $\begin{array}{c}\mathrm{Km} 185.3 \pm \\
68 \mu \mathrm{M}\end{array}$ & [61] \\
\hline$\left[{ }^{14} \mathrm{C}\right]$ colistin & $\begin{array}{l}\text { surface active agent which penetrates into and } \\
\text { disrupts the bacterial cell membrane; antibiotic } \\
\text { effective against most Gram-negative bacilli, } \\
\text { particularly Pseudomonas aeruginosa }\end{array}$ & hOCTN2 & $1 \mu \mathrm{M}$ & $\begin{array}{l}2.4 \mathrm{pmol} / \mathrm{mg} \\
\text { protein }\end{array}$ & $10 \mathrm{~min}$ & radioactive & & [67] \\
\hline$\left[{ }^{3} \mathrm{H}\right]$ etoposide & $\begin{array}{l}\text { DNA topoisomerase II inhibitor; treatment of } \\
\text { testicular cancer, small cell lung cancer, acute } \\
\text { myeloid leukaemia, lymphoma, ovarian cancer }\end{array}$ & hOCTN2 & ND & $2.85 \mu \mathrm{L} / \mathrm{mg} / 5 \mathrm{~min}$ & $5 \mathrm{~min}$ & radioactive & $\begin{array}{l}\text { independent of } \\
\mathrm{Na}+; \mathrm{Km} 150 \pm \\
\quad 34.1 \mu \mathrm{M}\end{array}$ & [62] \\
\hline mildronate & $\begin{array}{l}\text { inhibitor of gamma-butyrobetaine dioxygenase, } \\
\text { inhibits carnitine biosynthesis; } \\
\text { anti-ischemia medication }\end{array}$ & hOCTN2 & $10 \mu \mathrm{M}$ & $\begin{array}{l}\approx 250 \mu \mathrm{L} / \mathrm{min} \\
\text { protein }\end{array}$ & $1 \mathrm{~min}$ & LC/MS/MS & & [68] \\
\hline$\left[{ }^{14} \mathrm{C}\right]$ oxaliplatin & \multirow{2}{*}{$\begin{array}{l}\text { cross-links DNA, thus inhibiting DNA synthesis } \\
\text { and transcription; treatment of colon and } \\
\text { rectum advanced carcinoma }\end{array}$} & hOCTN2 & $1 \mu \mathrm{M}$ & $\begin{array}{l}\approx 1.5 \mu \mathrm{L} / \mathrm{mg} \\
\text { protein }\end{array}$ & $1 \mathrm{~h}$ & radioactive & & \multirow{2}{*}{ [63] } \\
\hline$\left[{ }^{14} \mathrm{C}\right]$ oxaliplatin & & rOctn2 & $1 \mu \mathrm{M}$ & $\begin{array}{l}\approx 3.5 \mu \mathrm{L} / \mathrm{mg} \\
\text { protein }\end{array}$ & $1 \mathrm{~h}$ & radioactive & & \\
\hline$\left[{ }^{3} \mathrm{H}\right]$ verapamil & $\begin{array}{c}\text { calcium channel blocker; class IV } \\
\text { anti-arrhythmia agent used to treat } \\
\text { hypertension, angina (chest pain), and certain } \\
\text { heart rhythm disorders }\end{array}$ & \multirow[t]{2}{*}{ mOctn2 } & $12 \mathrm{nM}$ & $275 \mu \mathrm{L} / \mathrm{mg}$ protein & $5 \mathrm{~min}$ & radioactive & & \multirow[t]{2}{*}[60]{} \\
\hline$\left[{ }^{3} \mathrm{H}\right]$ pyrilamine & H1 receptor; antihistamine & & $50 \mathrm{nM}$ & $118 \mu \mathrm{L} / \mathrm{mg}$ protein & $5 \mathrm{~min}$ & radioactive & & \\
\hline$\left[{ }^{3} \mathrm{H}\right]$ imatinib & $\begin{array}{c}\text { Bcr-Abl tyrosine-kinase inhibitor; cancer } \\
\text { treatment (chronic myelogenous leukemia } \\
\text { (CML), acute lymphoblastic leukemia (ALL), } \\
\text { gastrointestinal stromal tumors (GISTs) } \\
\text { and other) }\end{array}$ & mOctn2 & $0.2 \mu \mathrm{M}$ & $140 \%$ of control & ND & radioactive & & [64] \\
\hline
\end{tabular}


Table 3. Effect of drugs on inhibiting carnitine transport.

\begin{tabular}{|c|c|c|c|c|c|c|c|c|c|}
\hline \multirow{2}{*}{ Drug } & \multirow{2}{*}{ Drug Target and Use } & \multicolumn{3}{|c|}{ Concentration $\mu \mathrm{M}$} & \multicolumn{3}{|c|}{ Relative Uptake \% of Control } & \multirow{2}{*}{$\begin{array}{l}\text { Experimental } \\
\text { System }\end{array}$} & \multirow{2}{*}{ Ref. } \\
\hline & & hOCTN2 & rOctn2 & mOctn2 & hOCTN2 & rOctn2 & mOctn2 & & \\
\hline \multirow{2}{*}{ cephaloridine } & \multirow{2}{*}{$\begin{array}{l}\text { disrupts the synthesis of the } \\
\text { peptidoglycan layer of bacterial cell walls; } \\
\text { experimental drug, withdrawn from } \\
\text { clinical trials }\end{array}$} & 2500 & & & 10 & & & JAR; E & [69] \\
\hline & & 2500 & 2500 & & 13 & 25 & & HEK293; O & [69] \\
\hline \multirow{2}{*}{ cefepime } & \multirow{2}{*}{$\begin{array}{l}\text { disrupts the synthesis of the } \\
\text { peptidoglycan layer of bacterial cell walls; } \\
\text { active against Gram+ and Gram- bacteria, } \\
\text { for the treatment of pneumonia, urinary } \\
\text { tract infections, skin infections }\end{array}$} & 2500 & & & 19 & & & JAR; E & [69] \\
\hline & & 2500 & 2500 & & 27 & 28 & & HEK293; O & [69] \\
\hline \multirow{2}{*}{ cefluprenam } & \multirow{2}{*}{$\begin{array}{l}\text { disrupts the synthesis of the } \\
\text { peptidoglycan layer of bacterial cell walls; }\end{array}$} & 2500 & & & 58 & & & JAR; E & [69] \\
\hline & & 2500 & 2500 & & 74 & 76 & & HEK293; O & [69] \\
\hline $\begin{array}{l}\text { nelfinavir mesylate } \\
\text { hydrate }\end{array}$ & $\begin{array}{l}\text { HIV-1 protease inhibitor; antiviral drug } \\
\text { used for treatment of HIV }\end{array}$ & $11.87^{*}$ & $3.138^{*}$ & & 50 & 50 & & $\mathrm{CHO} ; \mathrm{O}$ & {$[70]$} \\
\hline \multirow{2}{*}{ ipratropium bromide } & \multirow{2}{*}{$\begin{array}{l}\text { blocks muscarinic acetylcholine receptors; } \\
\text { anticholinergic agent for treatment of } \\
\text { chronic obstructive pulmonary disease } \\
\text { (COPD) and asthma }\end{array}$} & 100 & & & $71.3 \pm 2.3$ & & & MDCKII; O & [71] \\
\hline & & $100 / 1000$ & & & $38 \pm 2 / 29 \pm 2$ & & & $\mathrm{~L} 6 ; \mathrm{O}$ & [72] \\
\hline nifedipine & $\begin{array}{l}\text { calcium channel blocker; used to treat } \\
\text { hypertension and chronic stable angina }\end{array}$ & $100 / 59.4^{*}$ & & & $69.6 \pm 16.4 / 50$ & & & MDCKII; O & [71] \\
\hline \multirow{2}{*}{ spironolactone } & \multirow{2}{*}{$\begin{array}{l}\text { antagonist of the mineralocorticoid } \\
\text { receptor; treatment of heart failure, } \\
\text { hyperaldosteronism, adrenal hyperplasia, } \\
\text { hypertension, and nephrotic syndrome }\end{array}$} & $100 / 36^{*}$ & & & $72.2 \pm 12.0 / 50$ & & & MDCKII; O & [71] \\
\hline & & 50 & & & $48 \pm 3$ & & & MDCKII; O & [73] \\
\hline $\begin{array}{l}\text { tetraethyl-ammonium } \\
\text { (TEA) }\end{array}$ & $\begin{array}{l}\text { blocks potassium and voltage-dependent } \\
\text { channels; no approved use in humans }\end{array}$ & $448.4 *$ & $211.8^{*}$ & & 50 & 50 & & $\mathrm{CHO} ; \mathrm{O}$ & [70] \\
\hline quinine & target not fully known; antimalarial drug & $26.94 *$ & $79.48^{*}$ & & 50 & 50 & & $\mathrm{CHO} ; \mathrm{O}$ & [70] \\
\hline \multirow{3}{*}{ quinidine } & \multirow{3}{*}{$\begin{array}{l}\text { blocker of voltage-gated sodium and } \\
\text { potassium channels; class I } \\
\text { antiarrhythmic agent }\end{array}$} & $17.44 *$ & $45.75^{*}$ & & 50 & 50 & & $\mathrm{CHO} ; \mathrm{O}$ & [70] \\
\hline & & & & 500 & & & $6.1 \pm 0.40$ & HEK293; O & [60] \\
\hline & & & & 500 & & & $\approx 36.4$ & $\mathrm{Nb2a} ; \mathrm{E}$ & [24] \\
\hline
\end{tabular}


Table 3. Cont.

\begin{tabular}{|c|c|c|c|c|c|c|c|c|c|}
\hline \multirow{2}{*}{ Drug } & \multirow{2}{*}{ Drug Target and Use } & \multicolumn{3}{|c|}{ Concentration $\mu \mathrm{M}$} & \multicolumn{3}{|c|}{ Relative Uptake \% of Control } & \multirow{2}{*}{$\begin{array}{l}\text { Experimental } \\
\text { System }\end{array}$} & \multirow{2}{*}{ Ref. } \\
\hline & & hOCTN2 & rOctn2 & mOctn2 & hOCTN2 & rOctn2 & mOctn2 & & \\
\hline \multirow{3}{*}{ lidocaine } & \multirow{3}{*}{$\begin{array}{l}\text { blocks sodium channels; local anesthetic, } \\
\text { also class Ib antiarrhythmic agent }\end{array}$} & & & 500 & & & $57.1 \pm 1.14$ & HEK293; O & [60] \\
\hline & & 100 & & & $77.6 \pm 7.8$ & & & MDCKII; O & [71] \\
\hline & & 50 & & & $69 \pm 7$ & & & MDCKII; O & [73] \\
\hline amiodarone & $\begin{array}{l}\text { blocker of voltage gated potassium and } \\
\text { voltage gated calcium channels; } \\
\text { antiarrhythmic drug }\end{array}$ & 100 & & & $66.3 \pm 16.2$ & & & MDCKII; O & [71] \\
\hline enalapril & $\begin{array}{l}\text { angiotensin-converting enzyme (ACE) } \\
\text { inhibitor; treatment of hypertension, heart } \\
\text { failure, asymptomatic left ventricular } \\
\text { dysfunction and diabetic nephropathy }\end{array}$ & 50 & & & $71 \pm 4$ & & & MDCKII; O & [73] \\
\hline \multirow{6}{*}{ verapamil } & \multirow{6}{*}{$\begin{array}{l}\text { calcium channel blocker; class IV } \\
\text { anti-arrhythmia agent used to treat } \\
\text { hypertension, angina (chest pain), and } \\
\text { certain heart rhythm disorders }\end{array}$} & $17.53 *$ & $46.66^{*}$ & & 50 & 50 & & $\mathrm{CHO} ; \mathrm{O}$ & [70] \\
\hline & & & & 500 & & & $1.3 \pm 0.12$ & HEK293; O & [60] \\
\hline & & & & 500 & & & $\approx 34$ & $\mathrm{Nb2a} ; \mathrm{E}$ & [24] \\
\hline & & $100 / 50.9 *$ & & & $66.5 \pm 38.3 / 50$ & & & MDCKII; O & [71] \\
\hline & & 50 & & & $58 \pm 2$ & & & MDCKII; O & [73] \\
\hline & & $100 / 1000$ & & & $54 \pm 1 / 28 \pm 1$ & & & L6; O & [72] \\
\hline simvastatine & $\begin{array}{l}\text { lipid-lowering drug; treatment of } \\
\text { dyslipidemia and to lower the risk of } \\
\text { cardiovascular disease }\end{array}$ & $8.457 *$ & $13.05^{*}$ & & 50 & 50 & & $\mathrm{CHO} ; \mathrm{O}$ & [70] \\
\hline \multirow{3}{*}{ pyrilamine } & \multirow{3}{*}{$\mathrm{H} 1$ receptor; antihistamine } & & & 500 & & & $15.4 \pm 0.73$ & HEK293; O & [60] \\
\hline & & 500 & & & $\approx 35$ & & & SW480; E & [74] \\
\hline & & 500 & & & $\approx 27.5$ & & & SW480; E & [74] \\
\hline diphenhydra-mine & $\begin{array}{l}\text { H1 receptor; antihistamine, also used for } \\
\text { tremor in parkinsonism and as antiemetic }\end{array}$ & & & 500 & & & $43.6 \pm 1.74$ & HEK293; O & [60] \\
\hline cortisone & $\begin{array}{l}\text { naturally occurring glucocorticoid; used } \\
\text { in replacement therapy for adrenal } \\
\text { insufficiency and as an } \\
\text { anti-inflammatory agent }\end{array}$ & 50 & & & $64 \pm 3$ & & & MDCKII; O & [73] \\
\hline
\end{tabular}


Table 3. Cont

\begin{tabular}{|c|c|c|c|c|c|c|c|c|c|}
\hline \multirow{2}{*}{ Drug } & \multirow{2}{*}{ Drug Target and Use } & \multicolumn{3}{|c|}{ Concentration $\mu \mathrm{M}$} & \multicolumn{3}{|c|}{ Relative Uptake \% of Control } & \multirow{2}{*}{$\begin{array}{c}\text { Experimental } \\
\text { System }\end{array}$} & \multirow{2}{*}{ Ref } \\
\hline & & hOCTN2 & rOctn2 & mOctn2 & hOCTN2 & rOctn2 & mOctn2 & & \\
\hline mildronate & $\begin{array}{l}\text { inhibitor of gamma-butyrobetaine } \\
\text { dioxygenase, inhibits carnitine } \\
\text { biosynthesis; anti-ischemia medication }\end{array}$ & 50 & & & $42 \pm 7$ & & & MDCKII; O & [73] \\
\hline rapamycin/sirolimus & $\begin{array}{l}\text { bind the cytosolic protein FK-binding } \\
\text { protein } 12 \text { (FKBP12) what inhibits the } \\
\text { mTOR kinase and blocks activation of } \mathrm{T} \\
\text { and B cells; immunosuppressive agent } \\
\text { indicated for the prevention of } \\
\text { transplant rejection }\end{array}$ & 100 & & 100 & $70.2 \pm 5.7$ & & $75.2 \pm 6.7$ & HEK293; O & [62] \\
\hline imatinib & $\begin{array}{l}\text { Bcr-Abl tyrosine-kinase inhibitor; cancer } \\
\text { treatment (chronic myelogenous leukemia } \\
\text { (CML), acute lymphoblastic leukemia } \\
\text { (ALL), gastrointestinal stromal tumors } \\
\text { (GISTs) and other) }\end{array}$ & $30.99 *$ & $71.19 *$ & & 50 & 50 & & $\mathrm{CHO} ; \mathrm{O}$ & {$[70]$} \\
\hline \multirow[t]{2}{*}{ vincristine } & \multirow{2}{*}{$\begin{array}{l}\text { binds tubulin and stops tubulin dimers } \\
\text { from polymerizing to form microtubules; } \\
\text { treatment of acute leukaemia, malignant } \\
\text { lymphoma, Hodgkin's disease, acute } \\
\text { erythraemia, and acute panmyelosis. }\end{array}$} & $39 *$ & $16.26^{*}$ & & 50 & 50 & & $\mathrm{CHO} ; \mathrm{O}$ & {$[70]$} \\
\hline & & 100 & & 100 & $33.6 \pm 0.1$ & & $101 \pm 7.3$ & HEK293; O & [62] \\
\hline paclitaxel & $\begin{array}{l}\text { hyper-stabilizes structure of polymerized } \\
\text { microtubules; treatment of ovarian cancer, } \\
\text { breast cancer, non-small cell lung } \\
\text { carcinoma, Kaposi sarcoma }\end{array}$ & 100 & & 100 & $64.9 \pm 1.8$ & & $29.3 \pm 8.1$ & HEK293; O & [62] \\
\hline daunorubicin & $\begin{array}{l}\text { intercalates DNA and inhibits } \\
\text { topoisomerase II activity; treatment of } \\
\text { acute nonlymphocytic leukemia } \\
\text { (myelogenous, monocytic, erythroid) }\end{array}$ & 50 & & 50 & $62.3 \pm 4.5$ & & $57.8 \pm 2.2$ & HEK293; O & [62] \\
\hline
\end{tabular}


Table 3. Cont.

\begin{tabular}{|c|c|c|c|c|c|c|c|c|c|}
\hline \multirow{2}{*}{ Drug } & \multirow{2}{*}{ Drug Target and Use } & \multicolumn{3}{|c|}{ Concentration $\mu \mathrm{M}$} & \multicolumn{3}{|c|}{ Relative Uptake \% of Control } & \multirow{2}{*}{$\begin{array}{c}\text { Experimental } \\
\text { System }\end{array}$} & \multirow{2}{*}{ Ref } \\
\hline & & hOCTN2 & rOctn2 & mOctn2 & hOCTN2 & rOctn2 & mOctn2 & & \\
\hline vinblastine & $\begin{array}{l}\text { binds to the microtubular proteins of the } \\
\text { mitotic spindle, preventing } \\
\text { polymerisation; treatment of Hodgkin's } \\
\text { disease, lymphocytic lymphoma, } \\
\text { advanced testicular cancer, Kaposi's } \\
\text { sarcoma, choriocarcinoma, breast } \\
\text { cancer, melanoma }\end{array}$ & 100 & & 100 & $58.4 \pm 1.1$ & & $79.9 \pm 10.4$ & HEK293; O & [62] \\
\hline sunitinib & $\begin{array}{l}\text { multi-targeted receptor tyrosine kinase } \\
\text { (RTK) inhibitor; treatment of renal cell } \\
\text { carcinoma (RCC) and imatinib-resistant } \\
\text { gastrointestinal stromal tumor (GIST) }\end{array}$ & 100 & & 100 & $46.2 \pm 1.4$ & & $63.6 \pm 9.5$ & HEK293; O & [62] \\
\hline etoposide & $\begin{array}{l}\text { DNA topoisomerase II inhibitor; } \\
\text { treatment of testicular cancer, small cell } \\
\text { lung cancer, acute myeloid leukaemia, } \\
\text { lymphoma, ovarian cancer }\end{array}$ & $100 / 55^{*}$ & & 100 & $41.2 \pm 4.5 / 50$ & & $64.3 \pm 4.1$ & HEK293; O & [62] \\
\hline \multirow{2}{*}{ vinorelbine } & $\begin{array}{l}\text { binds to tubulin and prevents formation } \\
\text { of the mitotic spindle treatment of }\end{array}$ & 100 & & 100 & $15.3 \pm 1.3$ & & $65.6 \pm 12.1$ & HEK293; O & [62] \\
\hline & $\begin{array}{l}\text { advanced nonsmall cell lung cancer } \\
\text { (NSCLC), metastatic breast cancer }\end{array}$ & 60 & & 60 & $\approx 20$ & & $\approx 65$ & HEK293; O & [75] \\
\hline cisplatin & $\begin{array}{l}\text { platinum-based chemotherapy drug that } \\
\text { intercalates DNA; treatment of various } \\
\text { types of cancers (e.g., small cell lung } \\
\text { cancer, metastatic testicular and ovarian } \\
\text { cancer, advanced bladder cancer, head } \\
\text { and neck epithelial tumors }\end{array}$ & 100 & & 100 & $\approx 90$ & & $\approx 65$ & HEK293; O & [75] \\
\hline
\end{tabular}


Table 3. Cont.

\begin{tabular}{|c|c|c|c|c|c|c|c|c|c|}
\hline \multirow{2}{*}{ Drug } & \multirow{2}{*}{ Drug Target and Use } & \multicolumn{3}{|c|}{ Concentration $\mu \mathrm{M}$} & \multicolumn{3}{|c|}{ Relative Uptake \% of Control } & \multirow{2}{*}{$\begin{array}{c}\text { Experimental } \\
\text { System }\end{array}$} & \multirow{2}{*}{ Ref. } \\
\hline & & hOCTN2 & rOctn2 & mOctn2 & hOCTN2 & rOctn2 & mOctn2 & & \\
\hline oxaliplatin & $\begin{array}{l}\text { cross-links DNA, thus inhibiting DNA } \\
\text { synthesis and transcription; treatment of } \\
\text { colon and rectum advanced carcinoma }\end{array}$ & 100 & & 100 & $\approx 85$ & & $\approx 100$ & HEK293; O & [75] \\
\hline cediranib & $\begin{array}{l}\text { Vascular endothelial growth factor } \\
\text { receptor-2 inhibitor; in development, } \\
\text { clinical trials for ovarian cancer, alveolar } \\
\text { soft part sarcoma, cervical cancer, } \\
\text { endometrial cancer, mesothelioma, } \\
\text { prostate cancer and solid tumors }\end{array}$ & $2.49 *$ & & & 50 & & & HEK293; O & [76] \\
\hline camptothecin (CZ112) & $\begin{array}{l}\text { selectively inhibits the nuclear enzyme } \\
\text { DNA topoisomerase, type I; investigated } \\
\text { for the treatment of cancer. }\end{array}$ & $\begin{array}{c}10 / 4.5 \pm \\
1.2\end{array}$ & & & $40 / 50$ & & & HEK293; O & [77] \\
\hline valporate & $\begin{array}{l}\text { not fully known; used to treat epilepsy } \\
\text { and bipolar disorder and to prevent } \\
\text { migraine headaches; has anti-proliferative } \\
\text { effects and is the subject of many clinical } \\
\text { trials in a variety of cancer types }\end{array}$ & 500 & & & $\approx 60$ & & & SW480; E & [74] \\
\hline
\end{tabular}




\section{SLC22A5 and Fatty Acid Oxidation in the Brain}

For many years, glucose has been considered the main energetic substrate of the adult brain [78,79], although lactate delivered by astrocytes was postulated to be an alternative energy source for activated neurons [80]. In 2003, Ebert et al. [81] demonstrated in nuclear magnetic resonance (NMR) studies that the brain of adult rat can oxidize ${ }^{13} \mathrm{C}$-octanoate, a process detected in astrocytes, and that this process accounts for $20 \%$ of the brain oxidative energy. Nonetheless, the utilization of fatty acids by the brain in energy delivery has remained controversial [82]. Oleate, a long-chain fatty acid, was suggested to cross the blood-brain barrier as well [83]. Moreover, the brain's ability to accumulate fatty acids was further evidenced through the detection of fatty acid transporters in the brain microvessel endothelial cells: fatty acid transport proteins (FATPs) 1 and 4, fatty acid binding protein 5 and fatty acid translocase/CD36 [84]. In 2017, Jernberg et al. [36] confirmed that four enzymes crucial for fatty acid oxidation-CPT1, CPT2 and two acyl-CoA dehydrogenases specific for either long-chain or medium-chain acyl-CoAs, as well as SLC22A5/OCTN2- are all expressed in various areas of the rat brain, with the expression of the last three even increasing throughout the development. The authors further demonstrated the capability of $\left[1-{ }^{14} \mathrm{C}\right]$ oleate oxidation in dissected cortex and hippocampus, as well as proved sensitivity of this process to etomoxir, an inhibitor of CPT1. Immunofluorescence experiments with the cell-type specific markers showed that CPT1a is found exclusively in astrocytes (GFAP-positive) and neural progenitor cells (Nestin-positive), although it was not detected in neurons, oligodendrocytes or microglia [36]. In addition, OCTN2 appears to be essential for the functioning of the brain; a deletion in SLC22A5 was reported in the attention deficit/hyperactivity disorder [85], and the deficiency of carnitine biosynthesis was revealed to be a risk factor in autism [86] with improvement after carnitine supplementation [87].

\section{SLC22A5 and Fatty Acid Oxidation in Cancer}

Interestingly, the fatty acid oxidation enzymes (including CPT1a) were also detected in glioma cells and the process of fatty acid oxidation contributed significantly to their aerobic respiration, while treatment with etomoxir decreased cell proliferation and prolonged survival in the mouse model of this disease [88]. Moreover, OCTN2 expression was increased in primary glioblastoma samples from patients, and even more so in samples from patients with recurrent glioblastoma, when compared to the healthy brain [89].

Metabolic reprogramming is now widely recognized as one of the so-called hallmarks of cancer, onset of traits, by which cells undergoing malignant transformation are characterized [90]. The uptake of glucose by cancer cells is high due to up-regulation of facilitative glucose transporter GLUT1 [91,92]. In cancer cells glucose is mainly metabolized in glycolysis and is preferentially used for synthesis of ribose, serine or protein glycosylation, which led to the hypothesis of malfunctioning of mitochondria (the so-called Warburg effect). Lactate, as an end product of glycolysis causes acidification of the microenvironment. Although researchers paid little attention to the process of fatty acid oxidation in the context of cancer cells, more and more papers are published which demonstrate that fatty acids that are taken up by cancer cells or come from hydrolyzed triglycerides can deliver ATP (for review, see [93]). The growth of cancer cells depends on the availability of NADPH, and the process of fatty acid oxidation delivers this co-enzyme for many anabolic processes [93]. Treatment of human glioblastoma cells with etomoxir, a CPT1 inhibitor, results in a decreased level of NADPH, depletion of ATP and an increased level of reactive oxygen species [94]. Furthermore, inhibition of fatty acid oxidation results in an increased level of cytotoxic lipids, such as proapoptotic ceramides containing palmitic and stearic acid, which in turn reduces mTOR signaling and rises the level of active caspase-3 [95]. What is more, cancer cells that oxidize fatty acids are resistant to radiation [96]. One needs to note that changes in cancer cells' metabolic pathways require transcriptional control. It was shown that in prostate cancer, among 10 co-regulators of a metabolic switch, the peroxisome proliferator-activated receptor gamma co-activator 1 alpha $(\mathrm{PGC} 1 \alpha)$-regulating the activity of estrogen-related receptor alpha (ERR $\alpha$ - -was identified, as well as that downregulation of PGC1 $\alpha$ promoted metastasis and 
disease progression [97]. PGC1 $\alpha$ was shown to induce transcription of several genes coding proteins involved in metabolic pathways, including those engaged in fatty acid catabolism [97]. Moreover, the activity of PGC1 $\alpha$-ERR $\alpha$ negatively correlated with levels of oncogenic transcription factor MYC [98]. However, it has to be added that the effect of PGC1 $\alpha$ differs across various tumors (for review, see [99]). A metabolomics analysis of triple-negative breast cancer cells with an elevated level of MYC demonstrated a four-fold increase in production of palmitoylcarnitine when compared to control mammary gland. The upregulation of many activators of fatty acid oxidation and the downregulation of many activators of fatty acid synthesis were also detected [100]. In addition, a decrease in $A C A C B$ coding acetyl-CoA carboxylase beta synthesising malonyl-CoA, a CPT1 inhibitor, was correlated with a worse prognosis in patients, while the proliferation rate of etomoxir-treated triple-negative cells was reduced [100].

All these reports give rise to a conclusion that cancer cells are dependent on lipid oxidation for their survival and proliferation; hence CPT1 is now recognized as a promising and potential target in anticancer therapy. However, available CPT1 inhibitors-etomoxir, oxfenicine and perhexeline-all cause side effects like neuropathy or hepatitis. Even though perhexeline is now an approved anti-angina agent in Australia, New Zealand and Asia, neither of the other two (etomoxir and oxfenicine) were successfully tried in human clinical trials, with etomoxir being even rejected from clinical trials due to the severity of hepatotoxic side effects [101]. Bearing in mind that CPT1 requires carnitine for its function, it is worth taking a closer look at SLC22A5, a transporter that delivers carnitine into the cells, in the context of cancer.

OCTN2 expression is altered in many types of cancer. It was shown to be down-regulated in virus and nonvirus-mediated epithelial cancers, and methylation of its promoter was proposed as a regulatory mechanism [102]. On the other hand, the expression of SLC22A5 was shown to be higher in high grade serous epithelial ovarian cancer [103]. An analysis with the use of CANCERTOOL [104] revealed that SLC22A5 expression was significantly lower in colorectal cancer than in normal tissue, which was also the case in the breast cancer. In lung adenocarcinoma, on the other hand, SLC22A5 expression was up-regulated when compared to the healthy lung.

A mutational analysis of metastatic breast cancer resulted in the identification of several driver mutations affecting transcription factors which regulate metastatic genes. SLC22A5 was among the mutated genes enhancing cancer cell migration [105]. This may be due to the presence of an intronic estrogen-response element (ERE) [38]. An analysis with the use of Human Protein Atlas (https://www.proteinatlas.org/humanproteome/pathology), a visualization tool for among other things The Cancer Genome Atlas (TCGA) datasets, demonstrates SLC22A5 expression in several cancers, including glioma, breast cancer and endometrial cancer, with the highest level of expression in renal cancer. The prognosis of overall survival correlated with SLC22A5 expression was either favorable or unfavorable, depending on the type of cancer (Table 4), with the biggest impact on patients' prognosis in pancreatic, renal and endometrial cancer.

Interestingly, a prognosis for breast cancer is better during the first 7 years for patients with a high SLC22A5 expression level; after this period, it is the other way round and a prognosis is much better in case of patients with a low expression level (Figure 4A). Another analysis of probability of overall survival in breast cancer patients with the use of KMplotter tool (https://kmplot.com/analysis/) revealed varying outcomes depending on which dataset and assay for gene expression data acquisition were employed. A dataset from PAN cancer expression data showed a similar prognosis to the one in Human Proteome Atlas (Figure 4B). However, the dataset with breast cancer patients' gene expression data collected with Affymetrix gene chip showed a prognosis for overall survival was significantly better for patients with high expression level of SLC22A5 (Figure 4C). Knowing from Wang et al. [38] that SLC22A5 expression is much higher in ER positive breast cancer than in ER negative one, also confirmed by Lu et al. [106], we further restricted this analysis to differentiate survival prognoses across $\mathrm{ER}+$ and $\mathrm{ER}$ - patients (Figure $4 \mathrm{E}, 4 \mathrm{~F}$ ). Interestingly, there was no significant difference in survival of the ER+ patients with high or low expression of SLC22A5. In the ER- patients, however, those with 
higher expression of SLC22A5 had a much better prognosis than those with low SLC22A5 expression and survival curve for the former was similar to those of ER+. This might mean that patients with breast cancer that have reached a certain threshold of SLC22A5 expression have a better prognosis regardless of ER status.

Table 4. Analysis of cancer patient survival correlated to SLC22A5 expression.

\begin{tabular}{|c|c|c|c|c|c|c|c|c|}
\hline \multicolumn{9}{|c|}{ Cancer Patient Survival Analysis Correlated to SLC22A5 Expression } \\
\hline \multirow{2}{*}{ Cancer Type } & \multirow{2}{*}{ Prognosis } & \multirow{2}{*}{$p$ Value } & \multicolumn{2}{|c|}{ \% 5 Year Survival } & \multicolumn{2}{|c|}{$\mathbf{n}$} & \multirow{2}{*}{$\begin{array}{c}\text { FPKM } \\
\text { Best Cut } \\
\text { Off }\end{array}$} & \multirow{2}{*}{$\begin{array}{c}\text { FPKM } \\
\text { Median }\end{array}$} \\
\hline & & & High & Low & High & Low & & \\
\hline glioma & unfavorable & 0.0046 & $6^{*}$ & $15^{*}$ & 105 & 48 & 1.21 & 1.51 \\
\hline melanoma & unfavorable & 0.16 & $22 *$ & $53 *$ & 48 & 54 & 0.72 & 0.71 \\
\hline thyroid cancer & unfavorable & 0.022 & 79 & 95 & 101 & 400 & 1.93 & 1.59 \\
\hline liver cancer & unfavorable & 0.015 & 42 & 63 & 257 & 108 & 0.69 & 0.87 \\
\hline prostate cancer & unfavorable & 0.035 & 97 & 99 & 283 & 211 & 3.07 & 3.27 \\
\hline ovarian cancer & unfavorable & 0.051 & 29 & 35 & 176 & 197 & 1.21 & 1.18 \\
\hline cervical cancer & unfavorable & 0.17 & 60 & 73 & 150 & 141 & 1.02 & 1.04 \\
\hline breast cancer & - & 0.39 & 86 & 77 & 600 & 475 & 2.54 & 2.73 \\
\hline pancreatic cancer & favorable & 0.00074 & 61 & 18 & 43 & 133 & 1.7 & 1.29 \\
\hline stomach cancer & favorable & 0.011 & 50 & 27 & 152 & 202 & 1.54 & 1.45 \\
\hline renal cancer & favorable & 0.00084 & 72 & 61 & 599 & 278 & 5.25 & 7.12 \\
\hline endometrial cancer & favorable & $2.9 \times 10^{-5}$ & 81 & 63 & 405 & 136 & 1.28 & 1.85 \\
\hline colorectal cancer & favorable & 0.022 & 67 & 51 & 411 & 188 & 1.49 & 1.78 \\
\hline head \& neck cancer & favorable & 0.12 & 52 & 41 & 208 & 291 & 0.81 & 0.75 \\
\hline testis cancer & favorable & 0.03 & 100 & 94 & 84 & 50 & 0.6 & 0.74 \\
\hline lung cancer & favorable & 0.092 & 45 & 45 & 647 & 347 & 0.92 & 1.06 \\
\hline urothelial cancer & favorable & 0.012 & 47 & 33 & 250 & 156 & 1.02 & 1.28 \\
\hline
\end{tabular}

The analysis performed on the data from Protein Atlas (https://www.proteinatlas.org/humanproteome/pathology). FPKM, Fragments per Kilobase of exon per Million reads, *3-years survival.

What is more, OCTN2 is ubiquitously expressed, including intestinal tract, hence it may play an important role in oral delivery of drugs. Due to a high affinity of L-carnitine for OCTN2, carnitine conjugates with several pharmacologically active compounds were tested in model systems to target some human diseases (for a review, see [107]). OCTN2 is also present in the blood brain barrier [29] and L-carnitine-conjugated poly(lactic-co-glycolytic acid) nanoparticles were shown to undergo transcytosis through this barrier and to be taken-up by glioma cells through OCTN2, which could improve the anti-glioma treatment [108].

In conclusion, L-carnitine is necessary for oxidation of fatty acids and is transported to the cells mainly by SLC22A5/OCTN2 protein, which is capable of transporting several drugs. This beneficial role of OCTN2 delivers energy to the cell. Fatty acid oxidation occurs not only in muscles and liver, but also in energy demanding cells like astrocytes and cancer cells, which can give an advantage to the latter by providing an alternative to the glucose source of energy for growth and proliferation. On the other hand, OCTN2 can transport several anti-cancer drugs leading to cell death. Moreover, OCTN2's very high affinity towards carnitine can be utilized for better drug delivery with the use of drug-carnitine conjugates or carnitine-coated particles. 
A. Human Protein Atlas RNA-seq

Overall Survival

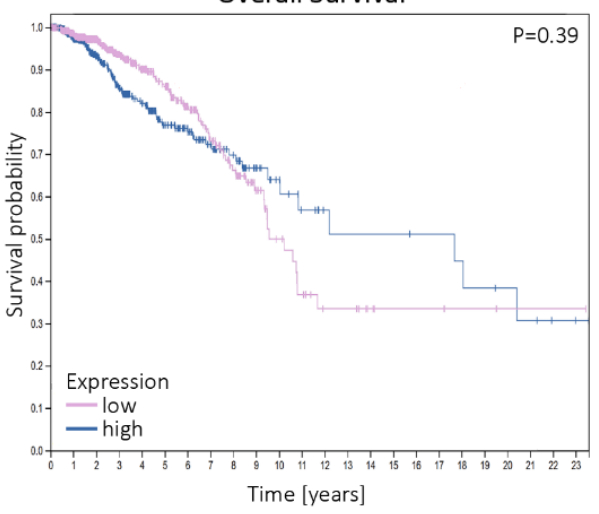

C.. KMplotter Breast Cancer dataset gene chip Overall Survival

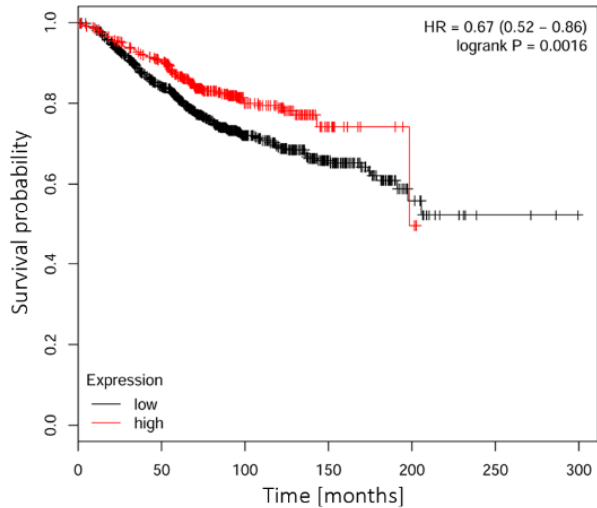

E. KMplotter Breast Cancer dataset gene chip; ER Overall Survival

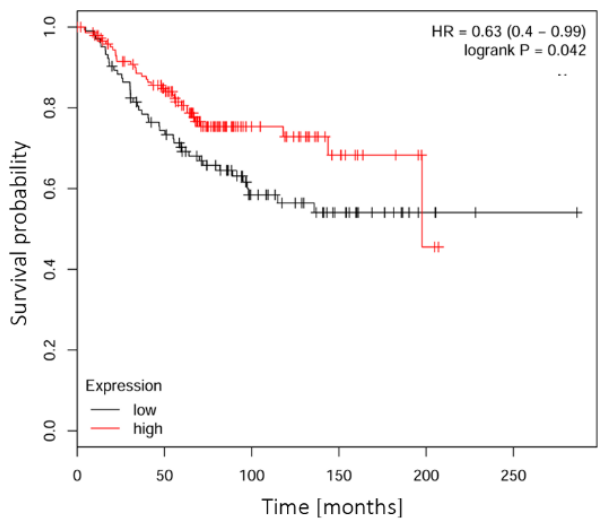

B. KMplotter PAN Cancer dataset RNA-seq

Overall Survival

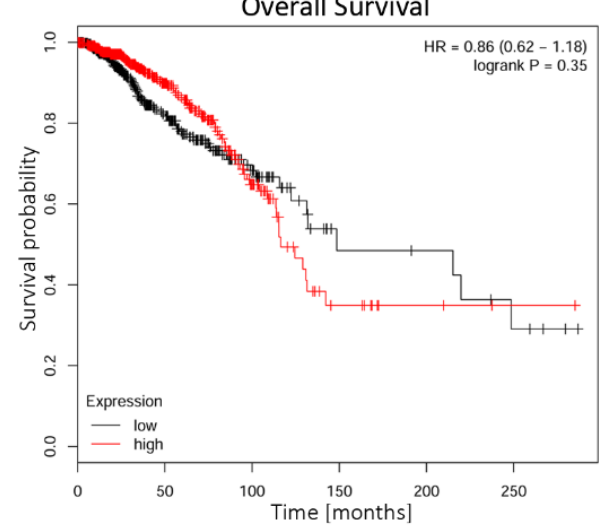

D.

CANCERTOOL Breast cancer dataset from Lu et al. SLC22A5 expression

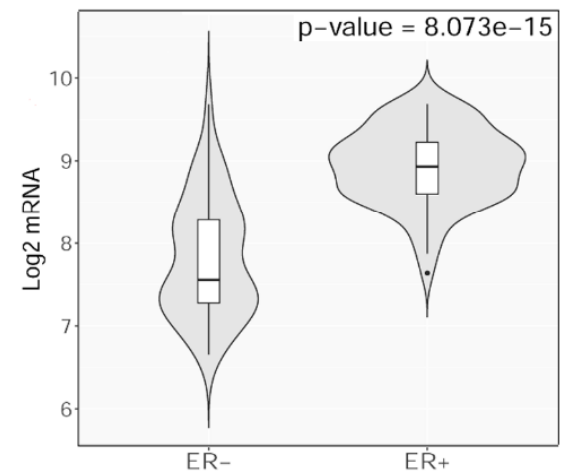

F. KMplotter Breast Cancer dataset gene chip; ER+ Overall Survival

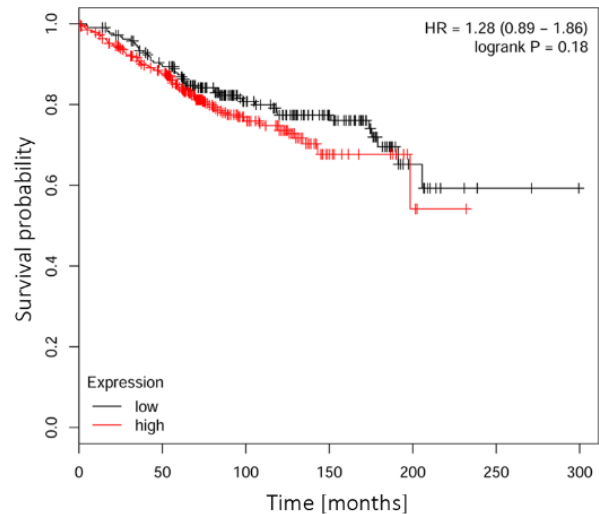

Figure 4. Probabulity of overall survival (OS) in breast cancer patients expressing high or low SLC22A5 levels. A. OS from Human protein Atlas with auto-selected best cutoff; mRNA data collected with RNA seq; B. OS assessed with the use of KMplotter and its PAN Cancer RNA seq dataset, with auto-selected best cutoff; C. OS assessed with the use of KMplotter and its Breast Cancer Affymetrix gene chip dataset, with auto-selected best cutoff; D. SLC22A5 expression in breast cancer ER- and ER+ samples from CANCERTOOL and dataset from [105]; E. OS assessed with the use of KMplotter and its Breast Cancer Affymetrix gene chip dataset restricted only to ER- patients, with auto-selected best cutoff; F. OS assessed with the use of KMplotter and its Breast Cancer Affymetrix gene chip dataset restricted only to ER+ patients, with auto-selected best cutoff. Graphic illustrations taken from proteinatlas.org, web.bioinformatics.cicbiogune.es/CANCERTOOL/and Kmplot.com. 
Author Contributions: B.J. and K.A.N. prepared the manuscript and approved the final manuscript as submitted. All authors have read and agreed to the published version of the manuscript.

Funding: This work was co-funded with National Science Centre in Poland grants 2018/31/B/NZ3/00669 (KAN) and 2016/23/N/NZ3/02430 (BJ).

Conflicts of Interest: The authors declare no conflict of interest.

\section{References}

1. Tein, I. Disorders of fatty acid oxidation. Handb. Clin. Neurol. 2013, 113, 1675-1688. [PubMed]

2. Wanders, R.J.; Waterham, H.R. Biochemistry of mammalian peroxisomes revisited. Annu. Rev. Biochem. 2006, 75, 295-332. [CrossRef] [PubMed]

3. Theodoulou, F.L.; Holdsworth, M.; Baker, A. Peroxisomal ABC transporters. FEBS Lett. 2006, 580, $1139-1155$. [CrossRef]

4. Palmieri, L.; Rottensteiner, H.; Girzalsky, W.; Scarcia, P.; Palmieri, F.; Erdmann, R. Identification and functional reconstitution of the yeast peroxisomal adenine nucleotide transporter. EMBO J. 2001, 20, 5049-5059. [CrossRef] [PubMed]

5. Lopez-Vinas, E.; Bentebibel, A.; Gurunathan, C.; Morillas, M.; de Arriaga, D.; Serra, D.; Asins, G.; Hegardt, F.G.; Gomez-Puertas, P. Definition by functional and structural analysis of two malonyl-CoA sites in carnitine palmitoyltransferase 1A. J. Biol. Chem. 2007, 282, 18212-18224. [CrossRef] [PubMed]

6. Kerner, J.; Hoppel, C. Fatty acid import into mitochondria. Biochim. Biophys. Acta 2000, 1486, 1-17. [CrossRef]

7. Faye, A.; Esnous, C.; Price, N.T.; Onfray, M.A.; Girard, J. Prip-Buus C: Rat liver carnitine palmitoyltransferase 1 forms an oligomeric complex within the outer mitochondrial membrane. J. Biol. Chem. 2007, 282, 26908-26916. [CrossRef]

8. Lee, K.; Kerner, J.; Hoppel, C.L. Mitochondrial carnitine palmitoyltransferase 1a (CPT1a) is part of an outer membrane fatty acid transfer complex. J. Biol. Chem. 2011, 286, 25655-25662. [CrossRef]

9. Indiveri, C.; Tonazzi, A.; Palmieri, F. Identification and purification of the carnitine carrier from rat liver mitochondria. Biochim. Biophys. Acta 1990, 1020, 81-86. [CrossRef]

10. Indiveri, C.; Iacobazzi, V.; Tonazzi, A.; Giangregorio, N.; Infantino, V.; Convertini, P.; Console, L.; Palmieri, F. The mitochondrial carnitine/acylcarnitine carrier: Function, structure and physiopathology. Mol. Aspects. Med. 2011, 32, 223-233. [CrossRef]

11. Ramsay, R.R. The role of the carnitine system in peroxisomal fatty acid oxidation. Am. J. Med. Sci. 1999, 318, 28-35. [CrossRef]

12. Januszewicz, E.; Pajak, B.; Gajkowska, B.; Samluk, L.; Djavadian, R.L.; Hinton, B.T.; Nalecz, K.A. Organic cation/carnitine transporter OCTN3 is present in astrocytes and is up-regulated by peroxisome proliferators-activator receptor agonist. Int. J. Biochem. Cell Biol. 2009, 41, 2599-2609. [CrossRef] [PubMed]

13. Vaz, F.M.; Wanders, R.J. Carnitine biosynthesis in mammals. Biochem. J. 2002, 361, 417-429. [CrossRef] [PubMed]

14. Van Vlies, N.; Wanders, R.J.; Vaz, F.M. Measurement of carnitine biosynthesis enzyme activities by tandem mass spectrometry: Differences between the mouse and the rat. Anal. Biochem. 2006, 354, 132-139. [CrossRef]

15. Steiber, A.; Kerner, J.; Hoppel, C.L. Carnitine: A nutritional, biosynthetic, and functional perspective. Mol. Aspects Med. 2004, 25, 455-473. [CrossRef]

16. Indiveri, C.; Giangregorio, N.; Iacobazzi, V.; Palmieri, F. Site-directed mutagenesis and chemical modification of the six native cysteine residues of the rat mitochondrial carnitine carrier: Implications for the role of cysteine-136. Biochemistry 2002, 41, 8649-8656. [CrossRef]

17. Tamai, I.; Ohashi, R.; Nezu, J.I.; Sai, Y.; Kobayashi, D.; Oku, A.; Shimane, M.; Tsuji, A. Molecular and functional characterization of organic cation/carnitine transporter family in mice. J. Biol. Chem. 2000, 275, 40064-40072. [CrossRef]

18. Lamhonwah, A.M.; Ackerley, C.A.; Tilups, A.; Edwards, V.D.; Wanders, R.J.; Tein, I. OCTN3 is a mammalian peroxisomal membrane carnitine transporter. Biochem. Biophy. Res. Co. 2005, 338, 1966-1972. [CrossRef]

19. Lamhonwah, A.M.; Hawkins, C.E.; Tam, C.; Wong, J.; Mai, L.; Tein, I. Expression patterns of the organic cation/carnitine transporter family in adult murine brain. Brain Dev. 2008, 30, 31-42. [CrossRef] 
20. Enomoto, A.; Wempe, M.F.; Tsuchida, H.; Shin, H.J.; Cha, S.H.; Anzai, N.; Goto, A.; Sakamoto, A.; Niwa, T.; Kanai, Y.; et al. Molecular identification of a novel carnitine transporter specific to human testis. Insights into the mechanism of carnitine recognition. J. Biol. Chem. 2002, 277, 36262-36271. [CrossRef]

21. Nakanishi, T.; Hatanaka, T.; Huang, W.; Prasad, P.D.; Leibach, F.H.; Ganapathy, M.E.; Ganapathy, V. Na+and $\mathrm{Cl}$-coupled active transport of carnitine by the amino acid transporter $\operatorname{ATB}(0,+)$ from mouse colon expressed in HRPE cells and Xenopus oocytes. J. Physiol. 2001, 532, 297-304. [CrossRef] [PubMed]

22. Tamai, I.; Ohashi, R.; Nezu, J.; Yabuuchi, H.; Oku, A.; Shimane, M.; Sai, Y.; Tsuji, A. Molecular and functional identification of sodium ion-dependent, high affinity human carnitine transporter OCTN2. J. Biol. Chem. 1998, 273, 20378-20382. [CrossRef] [PubMed]

23. Wu, X.; Prasad, P.D.; Leibach, F.H.; Ganapathy, V. cDNA sequence, transport function, and genomic organization of human OCTN2, a new member of the organic cation transporter family. Biochem. Biophy. Res. Co. 1998, 246, 589-595. [CrossRef] [PubMed]

24. Januszewicz, E.; Bekisz, M.; Mozrzymas, J.W.; Nalecz, K.A. High affinity carnitine transporters from OCTN family in neural cells. Neurochem. Res. 2010, 35, 743-748. [CrossRef] [PubMed]

25. Indiveri, C.; Iacobazzi, V.; Giangregorio, N.; Palmieri, F. The mitochondrial carnitine carrier protein: cDNA cloning, primary structure and comparison with other mitochondrial transport proteins. Biochem. J. 1997, 321, 713-719. [CrossRef]

26. Lamhonwah, A.M.; Skaug, J.; Scherer, S.W.; Tein, I. A third human carnitine/organic cation transporter (OCTN3) as a candidate for the 5q31 Crohn's disease locus (IBD5). Biochem. Biophy. Res. Co. 2003, 301, 98-101. [CrossRef]

27. Sloan, J.L.; Mager, S. Cloning and functional expression of a human $\mathrm{Na}(+)$ and $\mathrm{Cl}(-)$-dependent neutral and cationic amino acid transporter B(0+). J. Biol. Chem. 1999, 274, 23740-23745. [CrossRef]

28. Kido, Y.; Tamai, I.; Ohnari, A.; Sai, Y.; Kagami, T.; Nezu, J.; Nikaido, H.; Hashimoto, N.; Asano, M.; Tsuji, A. Functional relevance of carnitine transporter OCTN2 to brain distribution of L-carnitine and acetyl-L-carnitine across the blood- brain barrier. J. Neurochem. 2001, 79, 959-969. [CrossRef]

29. Berezowski, V.; Miecz, D.; Marszalek, M.; Broer, A.; Broer, S.; Cecchelli, R.; Nalecz, K.A. Involvement of OCTN2 and $\mathrm{B}^{0,+}$ in the transport of carnitine through an in vitro model of the blood-brain barrier. $J$. Neurochem. 2004, 91, 860-872. [CrossRef]

30. Michalec, K.; Mysiorek, C.; Kuntz, M.; Berezowski, V.; Szczepankiewicz, A.A.; Wilczynski, G.M.; Cecchelli, R.; Nalecz, K.A. Protein kinase C restricts transport of carnitine by amino acid transporter ATB $(0,+)$ apically localized in the blood-brain barrier. Arch. Biochem. Biophys. 2014, 554, 28-35. [CrossRef]

31. Wu, X.; Huang, W.; Prasad, P.D.; Seth, P.; Rajan, D.P.; Leibach, F.H.; Chen, J.; Conway, S.J.; Ganapathy, V. Functional characteristics and tissue distribution pattern of organic cation transporter 2 (OCTN2), an organic cation/carnitine transporter. J. Pharmacol. Exp. Ther. 1999, 290, 1482-1492. [PubMed]

32. Nezu, J.; Tamai, I.; Oku, A.; Ohashi, R.; Yabuuchi, H.; Hashimoto, N.; Nikaido, H.; Sai, Y.; Koizumi, A.; Shoji, Y.; et al. Primary systemic carnitine deficiency is caused by mutations in a gene encoding sodium ion-dependent carnitine transporter. Nat. Genet. 1999, 21, 91-94. [CrossRef] [PubMed]

33. Wu, X.; George, R.L.; Huang, W.; Wang, H.; Conway, S.J.; Leibach, F.H.; Ganapathy, V. Structural and functional characteristics and tissue distribution pattern of rat OCTN1, an organic cation transporter, cloned from placenta. Biochim. Biophys. Acta 2000, 1466, 315-327. [CrossRef]

34. Grundemann, D.; Harlfinger, S.; Golz, S.; Geerts, A.; Lazar, A.; Berkels, R.; Jung, N.; Rubbert, A.; Schomig, E. Discovery of the ergothioneine transporter. Proc. Natl. Acad. Sci. USA 2005, 102, 5256-5261. [CrossRef]

35. Cahoy, J.D.; Emery, B.; Kaushal, A.; Foo, L.C.; Zamanian, J.L.; Christopherson, K.S.; Xing, Y.; Lubischer, J.L.; Krieg, P.A.; Krupenko, S.A.; et al. A transcriptome database for astrocytes, neurons, and oligodendrocytes: A new resource for understanding brain development and function. J. Neurosci. 2008, 28, 264-278. [CrossRef]

36. Jernberg, J.N.; Bowman, C.E.; Wolfgang, M.J.; Scafidi, S. Developmental regulation and localization of carnitine palmitoyltransferases (CPTs) in rat brain. J. Neurochem. 2017, 142, 407-419. [CrossRef]

37. Ingoglia, F.; Visigalli, R.; Rotoli, B.M.; Barilli, A.; Riccardi, B.; Puccini, P.; Milioli, M.; Di Lascia, M.; Bernuzzi, G.; Dall'Asta, V. Human macrophage differentiation induces OCTN2-mediated L-carnitine transport through stimulation of mTOR-STAT3 axis. J. Leukoc. Biol. 2017, 101, 665-674. [CrossRef]

38. Wang, C.; Uray, I.P.; Mazumdar, A.; Mayer, J.A.; Brown, P.H. SLC22A5/OCTN2 expression in breast cancer is induced by estrogen via a novel intronic estrogen-response element (ERE). Breast Cancer Res. Treat. 2012, 134, 101-115. [CrossRef] 
39. Koch, A.; Konig, B.; Stangl, G.I.; Eder, K. PPAR alpha mediates transcriptional upregulation of novel organic cation transporters-2 and -3 and enzymes involved in hepatic carnitine synthesis. Exp. Biol. Med. 2008, 233, 356-365. [CrossRef]

40. Eder, K.; Ringseis, R. The role of peroxisome proliferator-activated receptor alpha in transcriptional regulation of novel organic cation transporters. Eur. J. Pharmacol. 2010, 628, 1-5. [CrossRef]

41. D’Argenio, G.; Petillo, O.; Margarucci, S.; Torpedine, A.; Calarco, A.; Koverech, A.; Boccia, A.; Paolella, G.; Peluso, G. Colon OCTN2 gene expression is up-regulated by peroxisome proliferator-activated receptor gamma in humans and mice and contributes to local and systemic carnitine homeostasis. J. Biol. Chem. 2010, 285, 27078-27087. [CrossRef] [PubMed]

42. Zhou, X.; Ringseis, R.; Wen, G.; Eder, K. Carnitine transporter OCTN2 and carnitine uptake in bovine kidney cells is regulated by peroxisome proliferator-activated receptor beta/delta. Acta Vet. Scand. 2014, 56, 21. [CrossRef] [PubMed]

43. Wen, G.; Ringseis, R.; Eder, K. Mouse OCTN2 is directly regulated by peroxisome proliferator-activated receptor alpha (PPARalpha) via a PPRE located in the first intron. Biochem. Pharmacol. 2009, 79, 768-776. [CrossRef] [PubMed]

44. Luo, H.; Zhang, Y.; Guo, H.; Zhang, L.; Li, X.; Ringseis, R.; Wen, G.; Hui, D.; Liang, A.; Eder, K.; et al. Transcriptional regulation of the human, porcine and bovine OCTN2 gene by PPARalpha via a conserved PPRE located in intron 1. BMC Genet. 2014, 15, 90. [CrossRef]

45. Zhou, X.; Ringseis, R.; Wen, G.; Eder, K. The pro-inflammatory cytokine tumor necrosis factor alpha stimulates expression of the carnitine transporter OCTN2 (novel organic cation transporter 2) and carnitine uptake via nuclear factor-kappaB in Madin-Darby bovine kidney cells. J. Dairy Sci. 2015, 98, 3840-3848. [CrossRef]

46. Nagai, K.; Takikawa, O.; Kawakami, N.; Fukao, M.; Soma, T.; Oda, A.; Nishiya, T.; Hayashi, M.; Lu, L.; Nakano, M.; et al. Cloning and functional characterization of a novel up-regulator, cartregulin, of carnitine transporter, OCTN2. Arch. Biochem. Biophys. 2006, 452, 29-37. [CrossRef]

47. Filippo, C.A.; Ardon, O.; Longo, N. Glycosylation of the OCTN2 carnitine transporter: Study of natural mutations identified in patients with primary carnitine deficiency. Biochim. Biophys. Acta 2011, 1812, 312-320. [CrossRef]

48. Czeredys, M.; Samluk, L.; Michalec, K.; Tulodziecka, K.; Skowronek, K.; Nalecz, K.A. Caveolin-1-a novel interacting partner of organic cation/carnitine transporter (octn2): Effect of protein kinase $C$ on this interaction in rat astrocytes. PLOS ONE 2013, 8, e82105. [CrossRef]

49. Rytting, E.; Audus, K.L. Contributions of phosphorylation to regulation of OCTN2 uptake of carnitine are minimal in BeWo cells. Biochem. Pharmacol. 2008, 75, 745-751. [CrossRef]

50. Kato, Y.; Sai, Y.; Yoshida, K.; Watanabe, C.; Hirata, T.; Tsuji, A. PDZK1 directly regulates the function of organic cation/carnitine transporter OCTN2. Mol. Pharmacol. 2005, 67, 734-743. [CrossRef]

51. Watanabe, C.; Kato, Y.; Sugiura, T.; Kubo, Y.; Wakayama, T.; Iseki, S.; Tsuji, A. PDZ adaptor protein PDZK2 stimulates transport activity of organic cation/carnitine transporter OCTN2 by modulating cell surface expression. Drug Metab. Dispos. 2006, 34, 1927-1934. [CrossRef] [PubMed]

52. Juraszek, B.; Nalecz, K.A. Protein phosphatase PP2A-a novel interacting partner of carnitine transporter OCTN2 (SLC22A5) in rat astrocytes. J. Neurochem. 2016, 139, 537-551. [CrossRef] [PubMed]

53. Jurkiewicz, D.; Michalec, K.; Skowronek, K.; Nałęcz, K.A. Tight junction protein ZO-1 controls organic cation/carnitine transporter OCTN2 (SLC22A5) in a protein kinase C-dependent way. Biochim. Biophys. Acta 2017, 1864, 797-805. [CrossRef] [PubMed]

54. Ye, F.; Zhang, M. Structures and target recognition modes of PDZ domains: Recurring themes and emerging pictures. Biochem. J. 2013, 455, 1-14. [CrossRef] [PubMed]

55. Hung, A.Y.; Sheng, M. PDZ domains: Structural modules for protein complex assembly. J. Biol. Chem. 2002, 277, 5699-5702. [CrossRef]

56. Herve, J.C.; Derangeon, M.; Sarrouilhe, D.; Bourmeyster, N. Influence of the scaffolding protein Zonula Occludens (ZOs) on membrane channels. Biochim. Biophys. Acta 2014, 1838, 595-604. [CrossRef]

57. Blumberg, P.M. Protein kinase $C$ as the receptor for the phorbol ester tumor promoters: Sixth Rhoads memorial award lecture. Cancer Res. 1988, 48, 1-8.

58. Seshacharyulu, P.; Pandey, P.; Datta, K.; Batra, S.K. Phosphatase: PP2A structural importance, regulation and its aberrant expression in cancer. Cancer Lett. 2013, 335, 9-18. [CrossRef] 
59. Wagner, C.A.; Lukewille, U.; Kaltenbach, S.; Moschen, I.; Broer, A.; Risler, T.; Broer, S.; Lang, F. Functional and pharmacological characterization of human $\mathrm{Na}(+)$ - carnitine cotransporter hOCTN2. Am. J. Physiol. Renal. Physiol. 2000, 279, 584-591. [CrossRef]

60. Ohashi, R.; Tamai, I.; Nezu, J.I.; Nikaido, H.; Hashimoto, N.; Oku, A.; Sai, Y.; Shimane, M.; Tsuji, A. Molecular and physiological evidence for multifunctionality of carnitine/organic cation transporter OCTN2. Mol. Pharmacol. 2001, 59, 358-366. [CrossRef]

61. Dos Santos Pereira, J.N.; Tadjerpisheh, S.; Abu Abed, M.; Saadatmand, A.R.; Weksler, B.; Romero, I.A.; Couraud, P.O.; Brockmoller, J.; Tzvetkov, M.V. The poorly membrane permeable antipsychotic drugs amisulpride and sulpiride are substrates of the organic cation transporters from the SLC22 family. AAPS J. 2014, 16, 1247-1258. [CrossRef] [PubMed]

62. Hu, C.; Lancaster, C.S.; Zuo, Z.; Hu, S.; Chen, Z.; Rubnitz, J.E.; Baker, S.D.; Sparreboom, A. Inhibition of OCTN2-mediated transport of carnitine by etoposide. Mol. Cancer Ther. 2012, 11, 921-929. [CrossRef] [PubMed]

63. Jong, N.N.; Nakanishi, T.; Liu, J.J.; Tamai, I.; McKeage, M.J. Oxaliplatin transport mediated by organic cation/carnitine transporters OCTN1 and OCTN2 in overexpressing human embryonic kidney 293 cells and rat dorsal root ganglion neurons. J. Pharmacol. Exp. Ther. 2011, 338, 537-547. [CrossRef] [PubMed]

64. Hu, S.; Franke, R.M.; Filipski, K.K.; Hu, C.; Orwick, S.J.; de Bruijn, E.A.; Burger, H.; Baker, S.D.; Sparreboom, A. Interaction of imatinib with human organic ion carriers. Clin. Cancer Res. 2008, 14, 3141-3148. [CrossRef]

65. Campone, M.; Berton-Rigaud, D.; Joly-Lobbedez, F.; Baurain, J.F.; Rolland, F.; Stenzl, A.; Fabbro, M.; van Dijk, M.; Pinkert, J.; Schmelter, T.; et al. A double-blind, randomized phase II study to evaluate the safety and efficacy of acetyl-L-carnitine in the prevention of sagopilone-induced peripheral neuropathy. Oncologist 2013, 18, 1190-1191. [CrossRef]

66. Hershman, D.L.; Unger, J.M.; Crew, K.D.; Minasian, L.M.; Awad, D.; Moinpour, C.M.; Hansen, L.; Lew, D.L.; Greenlee, H.; Fehrenbacher, L.; et al. Randomized double-blind placebo-controlled trial of acetyl-L-carnitine for the prevention of taxane-induced neuropathy in women undergoing adjuvant breast cancer therapy. $J$. Clin. Oncol. 2013, 31, 2627-2633. [CrossRef]

67. Visentin, M.; Gai, Z.; Torozi, A.; Hiller, C.; Kullak-Ublick, G.A. Colistin is substrate of the carnitine/organic cation transporter 2 (OCTN2, SLC22A5). Drug Metab. Dispos. 2017, 45, 1240-1244. [CrossRef]

68. Grigat, S.; Fork, C.; Bach, M.; Golz, S.; Geerts, A.; Schomig, E.; Grundemann, D. The carnitine transporter SLC22A5 is not a general drug transporter, but it efficiently translocates mildronate. Drug Metab. Dispos. 2009, 37, 330-337. [CrossRef]

69. Ganapathy, M.E.; Huang, W.; Rajan, D.P.; Carter, A.L.; Sugawara, M.; Iseki, K.; Leibach, F.H.; Ganapathy, V. Beta-lactam antibiotics as substrates for OCTN2, an organic cation/carnitine transporter. J. Biol. Chem. 2000, 275, 1699-1707. [CrossRef]

70. Szabo, K.; Nagy, Z.; Juhasz, V.; Zolnerciks, J.K.; Csorba, A.; Timar, Z.; Molnar, E.; Padar, P.; Johnson, W.; Beery, E.; et al. Species specificity profiling of rat and human organic cation/carnitine transporter Slc22a5/SLC22A5 (Octn2/OCTN2). Drug Metab. Pharmacokinet 2017, 32, 165-171. [CrossRef]

71. Grube, M.; Ameling, S.; Noutsias, M.; Kock, K.; Triebel, I.; Bonitz, K.; Meissner, K.; Jedlitschky, G.; Herda, L.R.; Reinthaler, M.; et al. Selective regulation of cardiac organic cation transporter novel type 2 (OCTN2) in dilated cardiomyopathy. Am. J. Pathol. 2011, 178, 2547-2559. [CrossRef] [PubMed]

72. Todesco, L.; Bur, D.; Brooks, H.; Torok, M.; Landmann, L.; Stieger, B.; Krahenbuhl, S. Pharmacological manipulation of L-carnitine transport into L6 cells with stable overexpression of human OCTN2. Cell Mol. Life Sci. 2008, 65, 1596-1608. [CrossRef] [PubMed]

73. Grube, M.; Meyer zu Schwabedissen, H.E.; Prager, D.; Haney, J.; Moritz, K.U.; Meissner, K.; Rosskopf, D.; Eckel, L.; Bohm, M.; Jedlitschky, G.; et al. Uptake of cardiovascular drugs into the human heart: Expression, regulation, and function of the carnitine transporter OCTN2 (SLC22A5). Circulation 2006, 113, 1114-1122. [CrossRef] [PubMed]

74. Elimrani, I.; Dionne, S.; Saragosti, D.; Qureshi, I.; Levy, E.; Delvin, E.; Seidman, E.G. Acetylcarnitine potentiates the anticarcinogenic effects of butyrate on SW480 colon cancer cells. Int. J. Oncol. 2015, 47, 755-763. [CrossRef] [PubMed]

75. Lancaster, C.S.; Hu, C.; Franke, R.M.; Filipski, K.K.; Orwick, S.J.; Chen, Z.; Zuo, Z.; Loos, W.J.; Sparreboom, A. Cisplatin-induced downregulation of OCTN2 affects carnitine wasting. Clin. Cancer Res. 2010, 16, 4789-4799. [CrossRef] 
76. Johnston, R.A.; Rawling, T.; Chan, T.; Zhou, F.; Murray, M. Selective inhibition of human solute carrier transporters by multikinase inhibitors. Drug Metab. Dispos. 2014, 42, 1851-1857. [CrossRef]

77. Zheng, J.; Chan, T.; Zhu, L.; Yan, X.; Cao, Z.; Wang, Y.; Zhou, F. The inhibitory effects of camptothecin (CPT) and its derivatives on the substrate uptakes mediated by human solute carrier transporters (SLCs). Xenobiotica 2016, 46, 831-840. [CrossRef]

78. Nehlig, A.; Pereira de Vasconcelos, A. Glucose and ketone body utilization by the brain of neonatal rats. Prog. Neurobiol. 1993, 40, 163-221. [CrossRef]

79. Patel, A.B.; Lai, J.C.; Chowdhury, G.M.; Hyder, F.; Rothman, D.L.; Shulman, R.G.; Behar, K.L. Direct evidence for activity-dependent glucose phosphorylation in neurons with implications for the astrocyte-to-neuron lactate shuttle. Proc. Natl. Acad. Sci. USA 2014, 111, 5385-5390. [CrossRef]

80. Pellerin, L.; Magistretti, P.J. Neuroenergetics: Calling upon astrocytes to satisfy hungry neurons. Neuroscientist 2004, 10, 53-62. [CrossRef]

81. Ebert, D.; Haller, R.G.; Walton, M.E. Energy contribution of octanoate to intact rat brain metabolism measured by 13C nuclear magnetic resonance spectroscopy. J. Neurosci. 2003, 23, 5928-5935. [CrossRef] [PubMed]

82. Schonfeld, P.; Reiser, G. Why does brain metabolism not favor burning of fatty acids to provide energy? Reflections on disadvantages of the use of free fatty acids as fuel for brain. J. Cereb. Blood Flow Metab. 2013, 33, 1493-1499. [CrossRef] [PubMed]

83. Mitchell, R.W.; Edmundson, C.L.; Miller, D.W.; Hatch, G.M. On the mechanism of oleate transport across human brain microvessel endothelial cells. J. Neurochem. 2009, 110, 1049-1057. [CrossRef] [PubMed]

84. Mitchell, R.W.; On, N.H.; Del Bigio, M.R.; Miller, D.W.; Hatch, G.M. Fatty acid transport protein expression in human brain and potential role in fatty acid transport across human brain microvessel endothelial cells. J. Neurochem. 2011, 117, 735-746. [CrossRef] [PubMed]

85. Lamhonwah, A.M.; Baric, I.; Lamhonwah, J.; Grubic, M.; Tein, I. Attention deficit/hyperactivity disorder as an associated feature in OCTN2 deficiency with novel deletion (p.T440-Y449). Clin. Case Rep. 2018, 6, 585-591. [CrossRef]

86. Celestino-Soper, P.B.; Violante, S.; Crawford, E.L.; Luo, R.; Lionel, A.C.; Delaby, E.; Cai, G.; Sadikovic, B.; Lee, K.; Lo, C.; et al. A common X-linked inborn error of carnitine biosynthesis may be a risk factor for nondysmorphic autism. Proc. Natl. Acad. Sci. USA 2012, 109, 7974-7981. [CrossRef]

87. Ziats, M.N.; Comeaux, M.S.; Yang, Y.; Scaglia, F.; Elsea, S.H.; Sun, Q.; Beaudet, A.L.; Schaaf, C.P. Improvement of regressive autism symptoms in a child with TMLHE deficiency following carnitine supplementation. Am. J. Med. Genet. A 2015, 167A, 2162-2167. [CrossRef]

88. Lin, H.; Patel, S.; Affleck, V.S.; Wilson, I.; Turnbull, D.M.; Joshi, A.R.; Maxwell, R.; Stoll, E.A. Fatty acid oxidation is required for the respiration and proliferation of malignant glioma cells. Neuro. Oncol. 2017, 19, 43-54. [CrossRef]

89. Fink, M.A.; Paland, H.; Herzog, S.; Grube, M.; Vogelgesang, S.; Weitmann, K.; Bialke, A.; Hoffmann, W.; Rauch, B.H.; Schroeder, H.W.S.; et al. L-Carnitine-Mediated Tumor Cell Protection and Poor Patient Survival Associated with OCTN2 Overexpression in Glioblastoma Multiforme. Clin. Cancer Res. 2019, 25, 2874-2886. [CrossRef]

90. Hanahan, D.; Weinberg, R.A. Hallmarks of cancer: The next generation. Cell 2011, 144, 646-674. [CrossRef]

91. Yamamoto, T.; Seino, Y.; Fukumoto, H.; Koh, G.; Yano, H.; Inagaki, N.; Yamada, Y.; Inoue, K.; Manabe, T.; Imura, H. Over-expression of facilitative glucose transporter genes in human cancer. Biochem. Biophys. Res. Commun. 1990, 170, 223-230. [CrossRef]

92. Yu, M.; Yongzhi, H.; Chen, S.; Luo, X.; Lin, Y.; Zhou, Y.; Jin, H.; Hou, B.; Deng, Y.; Tu, L.; et al. The prognostic value of GLUT1 in cancers: A systematic review and meta-analysis. Oncotarget 2017, 8, 43356-43367. [CrossRef] [PubMed]

93. Carracedo, A.; Cantley, L.C.; Pandolfi, P.P. Cancer metabolism: Fatty acid oxidation in the limelight. Nat. Rev. Cancer 2013, 13, 227-232. [CrossRef] [PubMed]

94. Pike, L.S.; Smift, A.L.; Croteau, N.J.; Ferrick, D.A.; Wu, M. Inhibition of fatty acid oxidation by etomoxir impairs NADPH production and increases reactive oxygen species resulting in ATP depletion and cell death in human glioblastoma cells. Biochim. Biophys. Acta 2011, 1807, 726-734. [CrossRef]

95. Schlaepfer, I.R.; Rider, L.; Rodrigues, L.U.; Gijon, M.A.; Pac, C.T.; Romero, L.; Cimic, A.; Sirintrapun, S.J.; Glode, L.M.; Eckel, R.H.; et al. Lipid catabolism via CPT1 as a therapeutic target for prostate cancer. Mol. Cancer Ther. 2014, 13, 2361-2371. [CrossRef] 
96. Harper, M.E.; Antoniou, A.; Villalobos-Menuey, E.; Russo, A.; Trauger, R.; Vendemelio, M.; George, A.; Bartholomew, R.; Carlo, D.; Shaikh, A.; et al. Characterization of a novel metabolic strategy used by drug-resistant tumor cells. FASEB J. 2002, 16, 1550-1557. [CrossRef]

97. Torrano, V.; Valcarcel-Jimenez, L.; Cortazar, A.R.; Liu, X.; Urosevic, J.; Castillo-Martin, M.; Fernandez-Ruiz, S.; Morciano, G.; Caro-Maldonado, A.; Guiu, M.; et al. The metabolic co-regulator PGC1alpha suppresses prostate cancer metastasis. Nat. Cell Biol. 2016, 18, 645-656. [CrossRef]

98. Valcarcel-Jimenez, L.; Macchia, A.; Crosas-Molist, E.; Schaub-Clerigue, A.; Camacho, L.; Martin-Martin, N.; Cicogna, P.; Viera-Bardon, C.; Fernandez-Ruiz, S.; Rodriguez-Hernandez, I.; et al. PGC1alpha suppresses prostate cancer cell invasion through ERRalpha transcriptional control. Cancer Res. 2019. [CrossRef]

99. Mastropasqua, F.; Girolimetti, G.; Shoshan, M. PGC1alpha: Friend or Foe in Cancer? Genes (Basel) 2018,9 , 48. [CrossRef]

100. Camarda, R.; Zhou, A.Y.; Kohnz, R.A.; Balakrishnan, S.; Mahieu, C.; Anderton, B.; Eyob, H.; Kajimura, S.; Tward, A.; Krings, G.; et al. Inhibition of fatty acid oxidation as a therapy for MYC-overexpressing triple-negative breast cancer. Nat. Med. 2016, 22, 427-432. [CrossRef]

101. Holubarsch, C.J.; Rohrbach, M.; Karrasch, M.; Boehm, E.; Polonski, L.; Ponikowski, P.; Rhein, S. A double-blind randomized multicentre clinical trial to evaluate the efficacy and safety of two doses of etomoxir in comparison with placebo in patients with moderate congestive heart failure: The ERGO (etomoxir for the recovery of glucose oxidation) study. Clin. Sci. 2007, 113, 205-212. [PubMed]

102. Scalise, M.; Galluccio, M.; Accardi, R.; Cornet, I.; Tommasino, M.; Indiveri, C. Human OCTN2 (SLC22A5) is down-regulated in virus- and nonvirus-mediated cancer. Cell Biochem. Funct. 2012, 30, 419-425. [CrossRef] [PubMed]

103. Elsnerova, K.; Mohelnikova-Duchonova, B.; Cerovska, E.; Ehrlichova, M.; Gut, I.; Rob, L.; Skapa, P.; Hruda, M.; Bartakova, A.; Bouda, J.; et al. Gene expression of membrane transporters: Importance for prognosis and progression of ovarian carcinoma. Oncol. Rep. 2016, 35, 2159-2170. [CrossRef] [PubMed]

104. Cortazar, A.R.; Torrano, V.; Martin-Martin, N.; Caro-Maldonado, A.; Camacho, L.; Hermanova, I.; Guruceaga, E.; Lorenzo-Martin, L.F.; Caloto, R.; Gomis, R.R.; et al. CANCERTOOL: A Visualization and Representation Interface to Exploit Cancer Datasets. Cancer Res. 2018, 78, 6320-6328. [CrossRef] [PubMed]

105. Lee, J.H.; Zhao, X.M.; Yoon, I.; Lee, J.Y.; Kwon, N.H.; Wang, Y.Y.; Lee, K.M.; Lee, M.J.; Kim, J.; Moon, H.G.; et al. Integrative analysis of mutational and transcriptional profiles reveals driver mutations of metastatic breast cancers. Cell Discov. 2016, 2, 16025. [CrossRef] [PubMed]

106. Lu, X.; Lu, X.; Wang, Z.C.; Iglehart, J.D.; Zhang, X.; Richardson, A.L. Predicting features of breast cancer with gene expression patterns. Breast Cancer Res. Treat. 2008, 108, 191-201. [CrossRef]

107. Kou, L.; Sun, R.; Ganapathy, V.; Yao, Q.; Chen, R. Recent advances in drug delivery via the organic cation/carnitine transporter 2 (OCTN2/SLC22A5). Expert Opin. Ther. Targets 2018, 22, 715-726. [CrossRef]

108. 108 Kou, L.; Hou, Y.; Yao, Q.; Guo, W.; Wang, G.; Wang, M.; Fu, Q.; He, Z.; Ganapathy, V.; Sun, J. L-Carnitine-conjugated nanoparticles to promote permeation across blood-brain barrier and to target glioma cells for drug delivery via the novel organic cation/carnitine transporter OCTN2. Artif. Cell Nanomed. B. 2018, 46, 1605-1616.

(C) 2019 by the authors. Licensee MDPI, Basel, Switzerland. This article is an open access article distributed under the terms and conditions of the Creative Commons Attribution (CC BY) license (http://creativecommons.org/licenses/by/4.0/). 